\title{
Area-Dependent Quantum Field Theory
}

\author{
Ingo Runkel ${ }^{1}$, Lóránt Szegedy ${ }^{2}$ (]) \\ ${ }^{1}$ Fachbereich Mathematik, Universität Hamburg, Bundesstraße 55, 20146 Hamburg, Germany. \\ E-mail: ingo.runkel@uni-hamburg.de \\ 2 Institute of Science and Technology Austria, Am Campus 1, 3400 Klosterneuburg, Austria. \\ E-mail: lorant.szegedy@ist.ac.at
}

Received: 23 November 2018 / Accepted: 24 August 2020

Published online: 20 November 2020 - (C) The Author(s) 2020

\begin{abstract}
Area-dependent quantum field theory is a modification of two-dimensional topological quantum field theory, where one equips each connected component of a bordism with a positive real number-interpreted as area — which behaves additively under glueing. As opposed to topological theories, in area-dependent theories the state spaces can be infinite-dimensional. We introduce the notion of regularised Frobenius algebras in Hilbert spaces and show that area-dependent theories are in one-to-one correspondence to commutative regularised Frobenius algebras. We also provide a state sum construction for area-dependent theories. Our main example is two-dimensional Yang-Mills theory with compact gauge group, which we treat in detail.
\end{abstract}

\section{Contents}

1. Introduction and Summary . . . . . . . . . . . . . . . . . . . 84

2. Regularised Frobenius Algebras . . . . . . . . . . . . . . . . 85

2.1 Definition of regularised algebras and Frobenius algebras . . . . . 86

2.2 Properties of RFAs . . . . . . . . . . . . . . . . . 90

2.3 Classification of Hermitian RFAs . . . . . . . . . . . . . . . . 93

2.4 Examples of RFAs . . . . . . . . . . . . . . . . . . . . . . . . . 97

3. Area-Dependent QFTs as Functors . . . . . . . . . . . . . . . . . . . . 99

3.1 Bordisms with area and aQFTs . . . . . . . . . . . . . . . 99

3.2 Equivalence of aQFTs and commutative RFAs . . . . . . . . . . . 101

4. State Sum Construction of aQFTs . . . . . . . . . . . . . . . . . . 101

4.1 PLCW decompositions with area . . . . . . . . . . . . . . 102

4.2 State sum construction . . . . . . . . . . . . . . . . . . . . 102

5. Example: 2d Yang-Mills Theory _. . . . . . . . . . . . . . . 107

5.1 Two RFAs from a compact group $G \ldots \ldots \ldots$

5.2 State sum construction of $2 \mathrm{~d}$ Yang-Mills theory . . . . . . . . . 112 
A Proof of Joint Continuity . . . . . . . . . . . . . . . . . . . . . . . . . . . . . . . . . . . . . . . 11

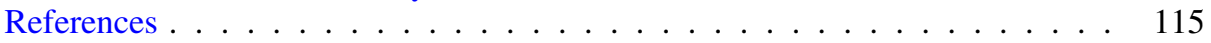

\section{Introduction and Summary}

Area-dependent quantum field theory $\left(\mathrm{aQFT}^{1}\right)$ is a modification of 2-dimensional topological quantum field theory (TQFT): we consider the category of bordisms with area $\operatorname{Bord}_{2}^{\text {area }}$ and symmetric monoidal functors from it to the category of Hilbert spaces $\mathcal{H} i l b$ which depend continuously on the area. We can think of the area as a positive number attached to each connected component of a surface, additive under composition. ${ }^{2}$ In order to have identities in $\mathcal{B o r d}_{2}^{\text {area }}$, we allow zero area on cylinders.

The main change when passing from TQFTs to aQFTs, and indeed the main motivation to look at this generalisation in the first place, is that the state spaces can now be infinite-dimensional. This is in contrast to TQFTs, where dualisability forces all state spaces to be finite-dimensional (see e.g. [CR, Sec. 2.4]). The same argument for aQFTs merely requires each state space to be a separable Hilbert space (cf. Lemma 2.9 and Theorem 3.5).

The main example of an aQFT is two-dimensional Yang-Mills theory for a compact semisimple Lie group $G$ [Mig, Rus, Wit], in which case the Hilbert space assigned to a circle is $C l^{2}(G)$, that is, square integrable class functions on $G$. We treat this example in detail in Sect. 5. Area-dependent theories in general have been considered in [Bru] and briefly in [Seg, Sec. 1.4] (see also [Bar, Sec. 4.5]). A construction of area-dependent theories using triangulations with equal triangle area has been given in [CTS].

Two-dimensional TQFTs are of course a special case of aQFT, namely they are aQFTs which are independent of the area parameters. Conversely one can show that if for all bordisms $\Sigma$ the zero area limit of $\mathcal{Z}(\Sigma)$ exists, then all state spaces $\mathcal{Z}(U)$ are necessarily finite dimensional, and the zero area limit of $\mathcal{Z}$ is a TQFT (Remark 3.6).

Recall that 2dTQFTs are in one-to-one correspondence to commutative Frobenius algebras [Dij, Abr], and that there is a state sum construction of 2dTQFTs which starts from a strongly separable symmetric (not necessarily commutative) Frobenius algebra $A$ as an input [BP,FHK,LP]. The commutative Frobenius algebra defining the TQFT obtained from this state sum construction is just the centre $Z(A)$.

The generalisation of these results to aQFTs is for the most part straightforward to the point of being mechanical: just add a positive real parameter to all maps in sight ("area parameters") and impose the condition that everything just depends on the sum of these areas.

The algebraic cornerstone of this work is the notion of a regularised Frobenius algebra (RFA), which consists of families of structure morphisms (product, unit, coproduct and counit), subject to the usual axioms of a Frobenius algebra, suitably decorated with area parameters (Definition 2.3).

An important example of an RFA is $L^{2}(G)$, the square integrable functions on a compact semisimple Lie group $G$. Here, the product $\mu_{a}$ and the coproduct $\Delta_{a}$ do have zero area limits given by the convolution product and by $\Delta_{0}(f)(g, h):=f(g h)$. The unit $\eta_{a}$ and counit $\varepsilon_{a}$ on the other hand do not have $a \rightarrow 0$ limits, see Sect. 5.1 for details. By the Peter-Weyl theorem we have $L^{2}(G)=\bigoplus_{V \in \widehat{G}} V \otimes V^{*}$, where the sum is a Hilbert

\footnotetext{
${ }^{1}$ We use the small ' $a$ ' in aQFT to set it apart from Algebraic QFT or Axiomatic QFT, which are often abbreviated as AQFT.

${ }^{2}$ This is equivalent to considering Riemann surfaces and remembering the induced volume form up to diffeomorphism, see [Mos,Ban].
} 
space direct sum over isomorphism classes of irreducible unitary representations of $G$, and the RFA structure on $L^{2}(G)$ restricts to an infinite direct sum of finite-dimensional RFAs on $V \otimes V^{*}$. This is a general result for Hermitian RFAs, i.e. RFAs for which $\mu_{a}^{\dagger}=\Delta_{a}$ and $\eta_{a}^{\dagger}=\varepsilon_{a}$ for every $a \in \mathbb{R}_{>0}$ (Theorem 2.19):

Theorem 1.1. Every Hermitian RFA is a Hilbert space direct sum of finite dimensional Hermitian RFAs.

All examples of non-hermitian RFAs we know are also direct sums of finite dimensional RFAs, but we are not aware of a proof that this holds in general (Remark 2.17).

Finite dimensional RFAs in turn are very simple: they are just usual Frobenius algebras $A$ together with an element $H$ in the centre $Z(A)$ of $A$, and the area-dependence is obtained by exponentiating $H$ (Corollary 2.15). This makes RFAs sound not very interesting, but note that, conversely, an infinite direct sum of finite-dimensional RFAs has to satisfy non-trivial bounds to again define an RFA (Proposition 2.16). And as the example of $L^{2}(G)$ shows, the direct sum decomposition may not always be the most natural perspective.

Our next main theorem generalises the classification of 2d TQFTs in terms of commutative Frobenius algebras as given in [Dij,Abr]. Namely, in Theorem 3.5 (and in Corollary 3.7) we show: ${ }^{3}$

Theorem 1.2. There is a one-to-one correspondence between (Hermitian) aQFTs and (Hermitian) commutative RFAs.

In Sect. 4.2 we furthermore generalise the state sum construction of [BP,FHK,LP]. We find that a strongly separable symmetric RFA $A$ (as defined in Sect. 2.1) providesunder one extra technical assumption - the data for the state sum construction of an aQFT, and the resulting aQFT corresponds, via Theorem 1.2, to the commutative RFA given by the centre of $A$.

This paper is organized as follows. In Sect. 2 we collect the algebraic preliminaries about RFAs, in Sect. 3 we state the definition of an aQFT and we show that aQFTs correspond to commutative RFAs. Section 4 contains the state sum construction and in Sect. 5 we give a detailed treatment of our main example, 2d YM theory.

\section{Regularised Frobenius Algebras}

In this section we study an algebraic notion-regularised Frobenius algebras - that will play a central role in characterising and producing examples of area-dependent QFTs. A more detailed treatment of this subject can be found in [Sze] where we also refer the reader for proofs that are omitted here. We denote with $\mathcal{H i l b}$ the category of Hilbert spaces and bounded linear maps with the strong operator topology on the hom sets $\mathcal{H i l b}(\mathcal{H}, \mathcal{K})=\mathcal{B}(\mathcal{H}, \mathcal{K})$.

\footnotetext{
3 In [Seg,Bar] the classification is instead in terms of algebras with a non-degenerate trace and an approximate unit. However, it is implicitly assumed there that the zero-area limit of the pair of pants with two in-going and one out-going boundary circles exists. This is not true for all examples as the commutative RFAs in Remark 2.25 illustrate.
} 


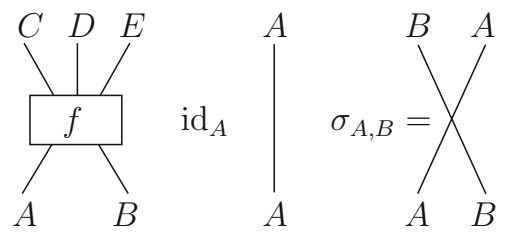

Fig. 1. Graphical notation of morphisms in the symmetric monoidal category Hilb. Here a morphism $f \in$ $\mathcal{B}(A \otimes B, C \otimes D \otimes E)$, the identity id $A \in \mathcal{B}(A, A)$ and the symmetric braiding $\sigma_{A, B}$ are shown. The tensor product of morphisms is depicted by drawing the morphisms next to each other and composition of morphisms is stacking them on top of each other

\subsection{Definition of regularised algebras and Frobenius algebras.}

Definition 2.1. A regularised algebra is an object $A \in \mathcal{H}$ ilb together with continuous families of morphisms

$$
\mu_{a} \in \mathcal{B}\left(A^{\otimes 2}, A\right) \text { and } \eta_{a} \in \mathcal{B}(\mathbb{C}, A)
$$

for every $a \in \mathbb{R}_{>0}$, called product and unit, such that the following relations hold:

1. for every $a, a_{1}, a_{2}, b_{1}, b_{2} \in \mathbb{R}_{>0}$, such that $a_{1}+a_{2}=b_{1}+b_{2}$,

$$
\begin{aligned}
& \mu_{a_{1}} \circ\left(\operatorname{id}_{A} \otimes \eta_{a_{2}}\right)=\mu_{b_{1}} \circ\left(\eta_{b_{2}} \otimes \mathrm{id}_{A}\right), \\
& \mu_{a_{1}} \circ\left(\operatorname{id}_{A} \otimes \mu_{a_{2}}\right)=\mu_{b_{1}} \circ\left(\mu_{b_{2}} \otimes \mathrm{id}_{A}\right) .
\end{aligned}
$$

2. Let $P_{a} \in \mathcal{B}(A, A)$ be given by (2.2), i.e. $P_{a}=\mu_{a_{1}} \circ\left(i d_{A} \otimes \eta_{a_{2}}\right)$ with $a=a_{1}+a_{2}$. We require that $\lim _{a \rightarrow 0} P_{a}=\mathrm{id}_{A}$.

Let $A, B \in \mathcal{H}$ ilb be regularised algebras. A morphism of regularised algebras $A \stackrel{f}{\rightarrow} B$ is a morphism in $\mathcal{H}$ ilb such that for every $a \in \mathbb{R}_{>0}$

$$
\eta_{a}^{B}=f \circ \eta_{a}^{A}, \quad \mu_{a}^{B} \circ(f \otimes f)=f \circ \mu_{a}^{A} .
$$

Instead of continuity of $\mu_{a}$ and $\eta_{a}$ in (2.1) it is enough to require continuity of $a \mapsto P_{a}$, for details see [Sze, Sec.4.1.1]. On the other hand, it is important not to impose the existence of an $a \rightarrow 0$ limit on $\mu_{a}$ and $\eta_{a}$; in Sect. 2.4 we will see examples where this limit does not exist, which would then have been excluded.

We will often use string diagram notation to represent morphisms in $\mathcal{H i l b}$, our conventions are given in Fig. 1. The morphisms in (2.1) are drawn as

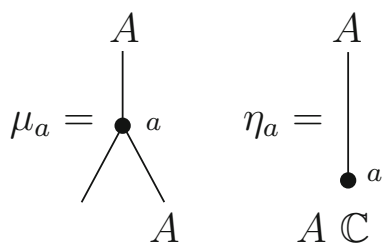


and the relations in (2.2) and (2.3) are

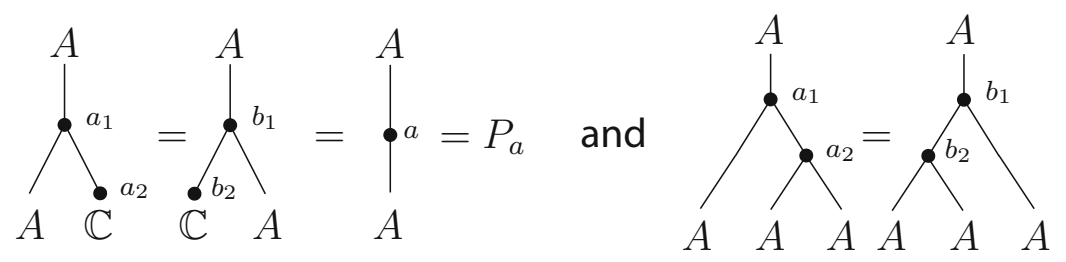

The next lemma gives some simple consequences of the above definition.

Lemma 2.2. Let $A$ be a regularised algebra. Let $a_{1}, a_{2}, b_{1}, b_{2}, c_{1}, c_{2} \in \mathbb{R}_{>0}$ such that $a_{1}+a_{2}=b_{2}+b_{2}=c_{1}+c_{2}$.

1. Let $\eta_{a}^{\prime} \in \mathcal{B}(\mathbb{C}, A)$ be a family of morphisms which satisfy (2.2) and Condition 2 of

Definition 2.1. Then $\eta_{a}^{\prime}=\eta_{a}$ for every $a \in \mathbb{R}_{>0}$.

2. $P_{a_{1}} \circ \eta_{a_{2}}=\eta_{a_{1}+a_{2}}$ and $P_{a_{1}} \circ P_{a_{2}}=P_{a_{1}+a_{2}}$.

3. $P_{a_{1}} \circ \mu_{a_{2}}=\mu_{b_{1}} \circ\left(P_{b_{2}} \otimes \mathrm{id}\right)=\mu_{c_{1}} \circ\left(\mathrm{id} \otimes P_{c_{2}}\right)=\mu_{a_{1}+a_{2}}$.

Proof. Here we only give the proof of Part 1, the proof of Part 2 and 3 are similar.

Let $a, b, c \in \mathbb{R}_{>0}$ and let us write $P_{a+b}^{\prime}:=\mu_{a} \circ\left(\eta_{b}^{\prime} \otimes \mathrm{id}_{A}\right)$ for the morphism in (2.2). From (2.2) we have that

$$
\mu_{a} \circ\left(\eta_{b} \otimes \eta_{c}^{\prime}\right)=\mu_{a} \circ\left(\eta_{c} \otimes \eta_{b}^{\prime}\right)
$$

as both sides only depend on the sum of the parameters. We then have that

$$
P_{a+b} \circ \eta_{c}^{\prime}=P_{a+b}^{\prime} \circ \eta_{c},
$$

and using that the composition is separately continuous together with $\lim _{a, b \rightarrow 0} P_{a+b}=$ $\lim _{a, b \rightarrow 0} P_{a+b}^{\prime}=\mathrm{id}_{A}$ we get that $\eta_{c}^{\prime}=\eta_{c}$ for every $c \in \mathbb{R}_{>0}$.

Next we introduce the dual concept to a regularised algebra. A regularised coalgebra is an object $A \in \mathcal{H} i l b$ together with continuous families of morphisms

$$
\Delta_{a}: A \rightarrow A^{\otimes 2} \text { and } \varepsilon_{a}: A \rightarrow \mathbb{C}
$$

for $a \in \mathbb{R}_{>0}$, called coproduct and counit, such that the following relations hold: for all $a, a_{1}, a_{2}, b_{1}, b_{2}>0$, such that $a_{1}+a_{2}=b_{1}+b_{2}=a$,

$$
\begin{aligned}
& \left(i d_{A} \otimes \varepsilon_{a_{2}}\right) \circ \Delta_{a_{1}}=\left(\varepsilon_{b_{2}} \otimes i d_{A}\right) \circ \Delta_{b_{1}}=: P_{a}^{\prime}, \\
& \left(i d_{A} \otimes \Delta_{a_{2}}\right) \circ \Delta_{a_{1}}=\left(\Delta_{b_{2}} \otimes i d_{A}\right) \circ \Delta_{b_{1}},
\end{aligned}
$$

and $\lim _{a \rightarrow 0} P_{a}^{\prime}=\mathrm{id}_{A}$. A morphism of regularised coalgebras, is a morphism of the objects which is compatible with $\Delta_{a}$ and $\varepsilon_{a}$ in the obvious way. Note that for a regularised coalgebra the dual statements of Lemma 2.2 hold. For the morphisms in (2.8) we introduce the following graphical notation:

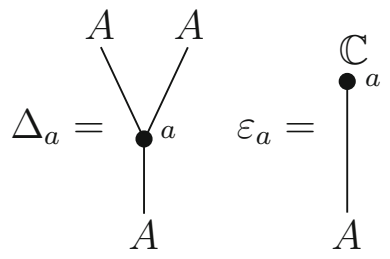

A key notion in this paper is the following: 
Definition 2.3. A regularised Frobenius algebra (or RFA in short) is a regularised algebra $A \in \mathcal{H}$ ilb, which is also a regularised coalgebra, such that

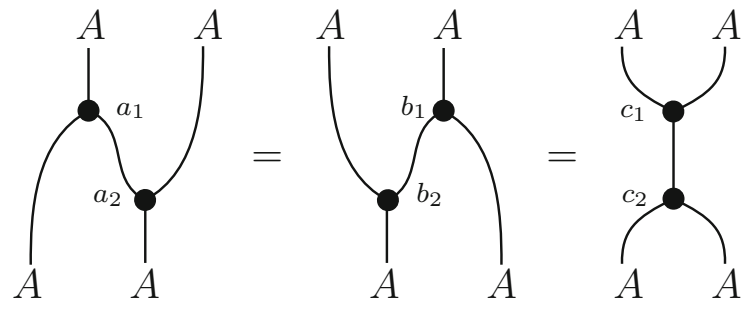

holds for all $a_{1}+a_{2}=b_{1}+b_{2}=c_{1}+c_{2}$. A morphism of RFAs, is a morphism of regularised algebras and coalgebras.

In an RFA $P_{a}$ from the algebra structure and $P_{a}^{\prime}$ from the coalgebra structure coincide:

Lemma 2.4. For an RFA we have $P_{a}=P_{a}^{\prime}$ for all $a>0$.

Proof. Let $a, b \in \mathbb{R}_{>0}$ be arbitrary. Choose $a_{1}, a_{2}, b_{2}, b_{2} \in \mathbb{R}_{>0}$ such that $a=a_{1}+a_{2}$, $b=b_{1}+b_{2}$ and $a>b_{1}$ and $b>a_{1}$ (e.g. $b_{1}=\frac{a}{2}, a_{1}=\frac{b}{2}$ ). By relation (2.12) one has that

$$
\left(\mu_{a_{2}} \otimes \operatorname{id}_{A}\right) \circ\left(\operatorname{id}_{A} \otimes \Delta_{b_{2}}\right)=\Delta_{a_{1}+a_{2}-b_{1}} \circ \mu_{b_{1}+b_{2}-a_{1}} .
$$

Composing (2.13) with id $A \otimes \varepsilon_{b_{1}}$ from the left and with $\eta_{a_{1}} \otimes \mathrm{id}_{A}$ from the right yields

$$
P_{a} \circ P_{b}^{\prime}=P_{a}^{\prime} \circ P_{b}
$$

We can take the $b \rightarrow 0$ limit on both sides of (2.14) and use separate continuity of the composition to get $P_{a}=P_{a}^{\prime}$.

We will prove the following lemma in "Appendix 5.2".

Lemma 2.5. In the monoidal sub-category of Hilb tensor generated by an RFA and its structure morphisms every morphism is jointly continuous in the parameters.

This lemma is not entirely immediate as composition in $\mathcal{H i l b}$ is only separately continuous but not jointly continuous in the strong operator topology, and e.g. the map $\mathcal{B}(H, H) \rightarrow \mathcal{B}(K \otimes H, K \otimes H), f \mapsto \mathrm{id} \otimes f$, is not continuous if $K, H$ are infinite dimensional.

Remark 2.6. Usual (non-regularised) Frobenius algebras have an equivalent characterisation via a non-degenerate invariant pairing. The same is true in the regularised setting, however as we will not need it here, we only refer the reader to [Sze].

Recall the symmetric braiding $\sigma$ on $\mathcal{H i l b}$. We call a regularised algebra $A \in \mathcal{H}$ ilb commutative if $\mu_{a} \circ \sigma_{A, A}=\mu_{a}$ for all $a \in \mathbb{R}_{>0}$. The centre of a regularised algebra $A$ is an object $B \in \mathcal{H}$ ilb and a morphism $i_{B}: B \rightarrow A$ such that

$$
\mu_{a} \circ \sigma_{A, A} \circ\left(i_{B} \otimes \mathrm{id}_{A}\right)=\mu_{a} \circ\left(i_{B} \otimes \mathrm{id}_{A}\right)
$$


for all $a \in \mathbb{R}_{>0}$, which is universal in the following sense. If there is an object $C$ and a morphism $f: C \rightarrow A$ satisfying the above equation then there is a unique morphism $\tilde{f}: C \rightarrow B$ such that the diagram

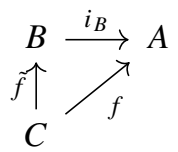

commutes. This implies in particular that $i_{B}$ is mono [Dav].

Lemma 2.7. The centre of a regularised algebra exists and it is a commutative regularised algebra.

Proof. One quickly checks that the closed subspace

$$
K:=\bigcap_{\substack{a \in \mathbb{R}_{>}>0 \\ x \in A}} \operatorname{ker}\left(\mu_{a}(x \otimes-)-\mu_{a}(-\otimes x)\right) \subseteq A
$$

satisfies (2.15). It satisfies the universal property (2.16) as any map $f: C \rightarrow A$ satisfying (2.15) lands in $K$.

Analogously to ordinary algebras we obtain induced product and unit morphisms on $K$ satisfying the algebraic conditions (2.2) and (2.3). Finally, that the map $\tilde{P}_{a}$ induced by $P_{a} \circ i_{K}=i_{K} \circ \tilde{P}_{a}$ satisfies $\lim _{a \rightarrow 0} \tilde{P}_{a}=\mathrm{id}_{K}$ follows from taking the $a \rightarrow 0$ limit on both sides of the defining equation and using that $i_{K}$ is an isometric embedding.

A regularised algebra is separable if there exists a family of morphisms $e_{a} \in$ $\mathcal{B}(\mathbb{C}, A \otimes A)$ for every $a \in \mathbb{R}_{>0}$ such that for $a_{1}+a_{2}=b_{1}+b_{2}=a$,

1. $\left(\mu_{a_{1}} \otimes \mathrm{id}_{A}\right) \circ\left(\mathrm{id}_{A} \otimes e_{a_{2}}\right)=\left(\mathrm{id}_{A} \otimes \mu_{b_{1}}\right) \circ\left(e_{b_{2}} \otimes \mathrm{id}_{A}\right)$ and

2. $\mu_{a_{1}} \circ e_{a_{2}}=\eta_{a}$.

The $e_{a}$ are called separability idempotents. A regularised algebra $A$ is strongly separable if it is separable and furthermore

3. $\sigma_{A, A} \circ e_{a}=e_{a}$.

These notions are direct generalisations of separability and strong separability for algebras, see e.g. [Kan,LP].

For an RFA $A$, we call the family of morphisms $\tau_{a}:=\mu_{a_{1}} \circ \Delta_{a_{2}} \circ \eta_{a_{3}}$ for $a_{1}, a_{2}, a_{3} \in$ $\mathbb{R}_{>0}$ with $a=a_{1}+a_{2}+a_{3}$ the window element of $A$, cf. [LP, Def. 2.12]. We call the window element invertible if there exists a family of morphisms $z_{a} \in \mathcal{B}(\mathbb{C}, A)$ for $a \in \mathbb{R}_{>0}$ (the inverse) such that $\mu_{a_{1}} \circ\left(\tau_{a_{2}} \otimes z_{a_{3}}\right)=\eta_{a_{1}+a_{2}+a_{3}}=\mu_{a_{1}} \circ\left(z_{a_{3}} \otimes \tau_{a_{3}}\right)$. From a direct computation one can verify that if there exists another family of morphisms $z_{a}^{\prime}$ which satisfies the above equation then $z_{a}^{\prime}=z_{a}$ for every $a \in \mathbb{R}_{>0}$, that is the inverse of the window element is unique. In the following we write $\tau_{a}^{-1}$ for the inverse of $\tau_{a}$. It is easy to check that the window element and its inverse satisfy (2.15).

An RFA is symmetric if $\varepsilon_{a_{1}} \circ \mu_{a_{2}} \circ \sigma=\varepsilon_{b_{1}} \circ \mu_{b_{2}}$. The following is a direct translation of [LP, Thm. 2.14] for strong separability for symmetric Frobenius algebras.

Proposition 2.8. A symmetric RFA is strongly separable if and only if its window element is invertible.

Proof. Set $e_{a}:=\Delta_{a_{1}} \circ \tau_{a_{2}}^{-1}$. Conversely set $\tau_{a}^{-1}:=\left(\varepsilon_{a_{1}} \otimes \mathrm{id}_{A}\right) \circ e_{a_{2}}$. 
2.2. Properties of RFAs. In this section we collect some properties of RFAs. The next lemma shows in particular that an RFA has a Hilbert basis with at most countably many elements.

Lemma 2.9. Let $A$ be an RFA.

1. The Hilbert space underlying $A$ is separable.

2. For all $a \in \mathbb{R}_{>0}, P_{a}$ is a trace class operator (and hence compact).

Proof. Part 1: Let $\left\{\phi_{j} \mid j \in I\right\}$ be a complete set of orthonormal vectors in $A$ and write $\gamma_{a}(1):=\Delta_{a_{1}} \circ \eta_{a_{2}}(1)=\sum_{k, l \in I} \phi_{k} \otimes \phi_{l} \gamma_{a}^{k l}$ with $a=a_{1}+a_{2}$. By [Kub, Cor. 5.28], independently of the countability of the indexing set $I$, there are at most countably many non-zero terms in the above sum. Thus for a given $a \in \mathbb{R}_{>0}$ there is a countable set of pairs $(k, l) \in I \times I$ such that $\gamma_{a}^{k l} \neq 0$. Define $I(a) \subseteq I$ to be the countable set of all elements of $I$ that appear in such a pair. Let

$$
J:=\bigcup_{n \in \mathbb{Z}_{>0}} I(1 / n) \subseteq I \quad \text { and } \quad A_{J}:=\overline{\operatorname{span}\left\{\phi_{j} \mid j \in J\right\}} \subseteq A
$$

Note that $J$ is countable and $A_{J}$ is separable. Write $\beta_{a}:=\varepsilon_{a_{1}} \circ \mu_{a_{2}}$. By (2.12), this satisfies

$$
\left(\operatorname{id}_{A} \otimes \beta_{a_{1}}\right) \circ\left(\gamma_{a_{2}} \otimes \operatorname{id}_{A}\right)=P_{a}
$$

and hence for every $v \in A$ and $n \in \mathbb{Z}_{>0}$ we have that

$$
P_{1 / n}(v) \in A_{J} \quad \text { and } \quad \lim _{n \rightarrow \infty} P_{1 / n}(v)=v,
$$

since $\lim _{n \rightarrow \infty} P_{1 / n}=\mathrm{id}_{A}$ in the strong operator topology. Since $A_{J}$ is closed, $v$ is an element of $A_{J}$. We have shown that $A_{J}=A$, and hence that $A$ is separable.

Part 2: First let us compute the following expression for some $a_{1}, a_{2} \in \mathbb{R}_{>0}$ :

$$
\beta_{a_{1}} \circ \sigma \circ \gamma_{a_{2}}(1)=\sum_{j, k \in I} \beta_{a_{1}}\left(\phi_{j} \otimes \phi_{k}\right) \gamma_{a_{2}}^{k j}
$$

This is an absolutely convergent sum, since the left hand side is a composition of bounded linear maps. We can rewrite this expression using (2.19) to get

$$
\begin{aligned}
\beta_{a_{1}} \circ \sigma \circ \gamma_{a_{2}}(1) & =\sum_{j, k, l \in I} \beta_{a_{1}}\left(\phi_{j} \otimes \phi_{k}\right) \gamma_{a_{2}}^{k l}\left\langle\phi_{j} \mid \phi_{l}\right\rangle \\
& \left.=\sum_{j, k, l \in I}\left\langle\phi_{j} \mid\left(\beta_{a_{1}} \otimes \mathrm{id}_{A}\right) \phi_{j} \otimes \phi_{k} \otimes \phi_{l} \gamma_{a_{2}}^{k l}\right\rangle\right\rangle \\
& \left.=\sum_{j \in I}\left\langle\phi_{j} \mid\left(\beta_{a_{1}} \otimes \mathrm{id}_{A}\right) \phi_{j} \otimes \sum_{k, l \in I} \phi_{k} \otimes \phi_{l} \gamma_{a_{2}}^{k l}\right\rangle\right\rangle \\
& =\sum_{j \in I}\left\langle\phi_{j} \mid\left(\beta_{a_{1}} \otimes \mathrm{id}_{A}\right) \circ\left(\mathrm{id}_{A} \otimes \gamma_{a_{2}}(1)\right) \phi_{j}\right\rangle \\
& =\sum_{j \in I}\left\langle\phi_{j} \mid P_{a} \phi_{j}\right\rangle,
\end{aligned}
$$


which is again an absolutely convergent sum. By [Con2, Ex. 18.2] $P_{a}$ is a trace class operator if and only if $\sum_{j \in I}\left\langle\phi_{j} \mid P_{a} \phi_{j}\right\rangle$ is absolutely convergent for every choice of orthonormal basis $\left\{\phi_{j}\right\}$, which we just have shown. In this case we have that

$$
\operatorname{tr}\left(P_{a}\right)=\sum_{j \in I}\left\langle\phi_{j} \mid P_{a} \phi_{j}\right\rangle .
$$

Let $A \in \mathcal{H}$ ilb be an RFA. By the Part 2 of Lemma 2.9 and [EN, Thm. II.4.29], $a \mapsto P_{a}$ (for $a>0$ ) is norm continuous. The following corollary shows that if we had defined Hilb to have the norm operator topology on hom-sets all examples of RFAs with self-adjoint $P_{a}$ would be finite-dimensional.

Corollary 2.10. Let $A \in \mathcal{H}$ Hilb be an RFA such that $\lim _{a \rightarrow 0} P_{a}=\operatorname{id}_{A}$ in the norm topology on $\mathcal{B}(A)$. Then $A$ is finite-dimensional.

Proof. From Lemma 2.9(2) we know that $P_{a}$ is compact for every $a \in \mathbb{R}_{>0}$. By [Con1, Prop. VI.3.4] the subspace of compact operators is closed in norm operator topology. These together with $\lim _{a \rightarrow 0} P_{a}=\mathrm{id}_{A}$ imply that $\mathrm{id}_{A}$ is compact, which in turn implies that $A$ is finite-dimensional.

We denote the category of RFAs by $\mathcal{R} \mathcal{F}$ rob and its subcategory of commutative RFAs by $c \mathcal{R} \mathcal{F}$ rob.

Proposition 2.11. Any morphism in $\mathcal{R} \mathcal{F}$ rob is mono and epi.

Proof. Let $\varphi: A \rightarrow B$ be a morphism of RFAs and let $\psi_{a, b}:=\left(\operatorname{id}_{A} \otimes \beta_{b}^{B}\right) \circ\left(\operatorname{id}_{A} \otimes \varphi \otimes\right.$ $\left.\operatorname{id}_{B}\right) \circ\left(\gamma_{a}^{A} \otimes \operatorname{id}_{B}\right)$. Then $\varphi \circ \psi_{a, b}=P_{a+b}^{B}$ and $\psi_{a, b} \circ \varphi=P_{a+b}^{A}$. We show that $\varphi$ is epi, showing that it is mono is similar. Let $f, g \in \mathcal{B}(B, X)$ for an object $X$ such that $f \circ \varphi=g \circ \varphi$. After composing with $\psi_{a, b}$ from the right for $a, b \in \mathbb{R}_{>0}$ we get $f \circ P_{a+b}^{B}=g \circ P_{a+b}^{B}$. This last equation holds for every $a, b \in \mathbb{R}_{>0}$, so we can take the limit $a, b \rightarrow 0$ to get $f=g$.

Remark 2.12. As we will see in Example 2 in Sect. 2.4, not every morphism of RFAs is invertible, hence $\mathcal{R} \mathcal{F}$ rob is not a groupoid.

For $A, B \in \mathcal{R} \mathcal{F}$ rob the object $A \otimes B$ is an RFAs by

$$
\begin{array}{ll}
\mu_{a}^{A \otimes B}:=\left(\mu_{a}^{A} \otimes \mu_{a}^{B}\right) \circ(\mathrm{id} \otimes \sigma \otimes \mathrm{id}), & \eta_{a}^{A \otimes B}:=\eta_{a}^{A} \otimes \eta_{a}^{B}, \\
\Delta_{a}^{A \otimes B}:=(\mathrm{id} \otimes \sigma \otimes \mathrm{id}) \circ\left(\Delta_{a}^{A} \otimes \Delta_{a}^{B}\right), & \varepsilon_{a}^{A \otimes B}:=\varepsilon_{a}^{A} \otimes \varepsilon_{a}^{B} .
\end{array}
$$

Proposition 2.13. $\mathcal{R} \mathcal{F}$ rob is a symmetric monoidal category with the above tensor product.

Recall that a Frobenius algebra in Hilb is always finite-dimensional see e.g. [Koc, Prop. 2.3.24].

Proposition 2.14. Let $A \in \mathcal{R} \mathcal{F}$ rob. The following are equivalent.

\section{A is finite-dimensional.}


2. All of the following limits exist:

$$
\lim _{a \rightarrow 0} \eta_{a}, \quad \lim _{a \rightarrow 0} \mu_{a}, \quad \lim _{a \rightarrow 0} \varepsilon_{a}, \quad \lim _{a \rightarrow 0} \Delta_{a} .
$$

Proof. $(1 \Rightarrow 2)$ : If $A$ is finite-dimensional, then the map $a \mapsto P_{a}$ is norm continuous, hence $P_{a}=e^{a H}$ for some $H \in \mathcal{B}(A)$. Then $\eta_{0}:=e^{-a H} \eta_{a}$ is independent of $a$ and $\eta_{a}=P_{a} \circ \eta_{0}$, hence $\lim _{a \rightarrow 0} \eta_{a}=\eta_{0}$ exists. One similarly proves that the other limits exist as well.

$(1 \Leftarrow 2)$ : The morphisms given by these limits define a Frobenius algebra structure on $A$, hence $A$ is finite-dimensional.

Corollary 2.15. A finite dimensional RFA A is in fact an ordinary Frobenius algebra $A^{(0)}$ together with an element in its centre $H \in Z\left(A^{(0)}\right)$ which one obtains by differentiating $P_{a}$ at $a=0$. Conversely, the area depenence on $A^{(0)}$ can be restored by setting $P_{a}:=$ $e^{a H}$.

For more details on this, see [Sze, Sec.4.1.4].

Proposition 2.16. Let I be a countable (possibly infinite) set. For $k \in I$ let $F_{k} \in \mathcal{H i l b}$ be a (possibly infinite-dimensional) RFA. Then $\bigoplus_{k \in I} F_{k}$ (the completed direct sum of Hilbert spaces) is an RFA if and only if, for every $a \in \mathbb{R}_{>0}$,

$$
\begin{aligned}
& \sup _{k \in I}\left\|\mu_{a}^{k}\right\|<\infty \text { and } \sup _{k \in I}\left\|\Delta_{a}^{k}\right\|<\infty, \\
& \sum_{k \in I}\left\|\varepsilon_{a}^{k}\right\|^{2}<\infty \text { and } \sum_{k \in I}\left\|\eta_{a}^{k}\right\|^{2}<\infty,
\end{aligned}
$$

where $\mu_{a}^{k}, \Delta_{a}^{k}, \varepsilon_{a}^{k}$ and $\eta_{a}^{k}$ denote the structure maps of $F_{k}$.

Proof. Let $F:=\bigoplus_{k \in I} F_{k}$ and fix the value of $a$.

$(\Rightarrow)$ : Let us write $x_{k}$ for the $k$ 'th component of $x \in F=\bigoplus_{k \in I} F_{k}$. Then for every $k \in I$

$$
\left\|\Delta_{a}^{k}\right\|=\sup _{\substack{x_{k} \in F_{k} \\\left\|x_{k}\right\|=1}}\left\|\Delta_{a}^{k}\left(x_{k}\right)\right\|=\sup _{\substack{x_{k} \in F_{k} \\\left\|x_{k}\right\|=1}}\left\|\Delta_{a}\left(x_{k}\right)\right\| \leq \sup _{\substack{x_{k} \in F_{k} \\\left\|x_{k}\right\|=1}}\left\|\Delta_{a}\right\| \cdot\left\|x_{k}\right\|=\left\|\Delta_{a}\right\|<\infty,
$$

so in particular $\sup _{k}\left\|\Delta_{a}^{k}\right\|<\infty$. A similar proof applies to the case of $\mu_{a}$. We calculate the norm of $\eta_{a}$ :

$$
\left\|\eta_{a}\right\|^{2}=\left\|\eta_{a}(1)\right\|^{2}=\sum_{k \in I}\left\|\eta_{a}^{k}(1)\right\|^{2}=\sum_{k \in I}\left\|\eta_{a}^{k}\right\|^{2}
$$

which is finite if and only if $\eta_{a}$ is a bounded operator. If $\varepsilon_{a}$ is bounded, then by the Riesz Lemma there exists a unique $v \in F$ such that $\varepsilon_{a}(x)=\langle v, x\rangle$ and $\left\|\varepsilon_{a}\right\|=\|v\|$. Then $\left\langle v_{k}, x_{k}\right\rangle=\left\langle v, x_{k}\right\rangle=\varepsilon_{a}\left(x_{k}\right)=\varepsilon_{a}^{k}\left(x_{k}\right)$. So again by the Riesz Lemma $\left\|\varepsilon_{a}^{k}\right\|=\left\|v_{k}\right\|$. We have that

$$
\left\|\varepsilon_{a}\right\|^{2}=\|v\|^{2}=\sum_{k \in I}\left\|v_{k}\right\|^{2}=\sum_{k \in I}\left\|\varepsilon_{a}^{k}\right\|^{2}
$$


$(\Leftarrow)$ : The operators $\eta_{a}$ and $\varepsilon_{a}$ are bounded by the previous discussion. For $\Delta_{a}$ one has that

$$
\begin{aligned}
\left\|\Delta_{a}\right\|^{2} & =\sup _{\substack{x \in F \\
\|x\|=1}}\left\|\Delta_{a}(x)\right\|^{2}=\sup _{\substack{x \in F \\
\|x\|=1}}\left\|\sum_{k \in I} \Delta_{a}\left(x_{k}\right)\right\|^{2}=\sup _{\substack{x \in F \\
\|x\|=1}} \sum_{k \in I}\left\|\Delta_{a}^{k}\left(x_{k}\right)\right\|^{2} \\
& \leq \sup _{\substack{x \in F \\
\|x\|=1}} \sum_{k \in I}\left\|\Delta_{a}^{k}\right\|^{2}\left\|x_{k}\right\|^{2} \leq\left(\sup _{l}\left\|\Delta_{a}^{l}\right\|^{2}\right) \cdot \sup _{\substack{x \in F \\
\|x\|=1}} \sum_{k \in I}\left\|x_{k}\right\|^{2}=\sup _{l}\left\|\Delta_{a}^{l}\right\|^{2}<\infty,
\end{aligned}
$$

so $\Delta_{a}$ is bounded. For $\mu_{a}$ the proof is similar.

Then one needs to check that $a \mapsto P_{a}:=\sum_{k \in I} P_{a}^{k}$ is continuous. Let $\varepsilon \in \mathbb{R}_{>0}$, $a_{0} \in \mathbb{R}_{\geq 0}$ and $f \in \bigoplus_{k \in I} F_{k}$ with components $f_{k}$ be fixed. Let $a^{\prime}>a_{0}$ and $0<E<\varepsilon$ be arbitrary. Since $P_{a}-P_{a_{0}}$ is a bounded operator, one can find $J_{a^{\prime}} \subset I$ finite, such that for every $a<a^{\prime}$

$$
\sum_{j \in I \backslash J_{a^{\prime}}}\left\|\left(P_{a}^{j}-P_{a_{0}}^{j}\right) f_{j}\right\|^{2}<E .
$$

Then let $\delta^{\prime}>0$ be such that for every $\left|a-a_{0}\right|<\delta^{\prime}$

$$
\sum_{j \in J_{a^{\prime}}}\left\|\left(P_{a}^{j}-P_{a_{0}}^{j}\right) f_{j}\right\|^{2}<\varepsilon-E,
$$

which can be chosen since the sum is finite and each $P_{a}^{j}$ is continuous by assumption. Finally let $\delta:=\min \left\{\delta^{\prime}, a^{\prime}-a_{0}\right\}$. By construction we have that for every $\left|a-a_{0}\right|<\delta$,

$$
\left\|\left(P_{a}-P_{a_{0}}\right) f\right\|^{2}=\sum_{j \in I}\left\|\left(P_{a}^{j}-P_{a_{0}}^{j}\right) f_{j}\right\|^{2}<\varepsilon .
$$

Remark 2.17. All examples of RFAs known to us are of the form given in Proposition 2.16 with finite-dimensional summands $F_{k}$. In fact we can show from the statements in [BB, Sec. 6.4] that all RFAs are (not necessarily orthogonal) direct sums of finite dimensional RFAs and a direct summand, on which $P_{a}$ is quasinilpotent for every $a>0$. This summand may be either 0 or infinite dimensional. The finite dimensional summands are generalised eigenspaces of $P_{a}$ for every $a>0$, while the remaining factor is the intersection of their complement. On the latter $P_{a}$ is quasinilpotent for every $a>0$ by [BB, Cor. 7.2.4]. For Hermitian RFAs, which we will introduce in Sect. 2.3, we show that this direct summand is 0 (see the proof of Theorem 2.19). We do not know an example of an RFA where this summand is non-zero. Note also that the same RFA $F_{k}$ cannot appear infinitely many times, as otherwise the bounds (2.26) would be violated.

2.3. Classification of Hermitian RFAs. We start by recalling the notion of a dagger (or $\dagger$-) symmetric monoidal category $\mathcal{S}$, e.g. from [Sel]. A dagger structure on $\mathcal{S}$ is a functor $(-)^{\dagger}: \mathcal{S} \rightarrow \mathcal{S}^{o p p}$ which is identity on objects, $(-)^{\dagger \dagger}=\operatorname{id}_{\mathcal{S}},(f \otimes g)^{\dagger}=f^{\dagger} \otimes g^{\dagger}$ for any morphisms $f, g$ and $\sigma_{U, V}^{\dagger}=\sigma_{V, U}$. We fix the dagger structure on $\mathcal{H i l b}$ given by adjoints. 
Definition 2.18. A Hermitian regularised Frobenius algebra (or $\dagger-\mathrm{RFA}$ for short) is an RFA for which $\mu_{a}^{\dagger}=\Delta_{a}$ and $\eta_{a}^{\dagger}=\varepsilon_{a}$ (and therefore $P_{a}=P_{a}^{\dagger}$ ). We denote by $\dagger-\mathcal{R} \mathcal{F}$ rob the full subcategory of $\mathcal{R} \mathcal{F}$ rob of $\dagger$-RFAs.

Let $\uparrow-\mathcal{F} r o b^{\mathrm{F}}$ denote the category which has objects countable families $\Phi=\left\{F_{j}, \sigma_{j}\right\}_{j \in I}$ of $\dagger$-Frobenius algebras $F_{j}$ and real numbers $\sigma_{j}$, such that for every $a \in \mathbb{R}_{>0}$

$$
\sup _{j \in I}\left\{e^{-a \sigma_{j}}\left\|\mu_{j}\right\|\right\}<\infty \text { and } \sum_{j \in I} e^{-2 a \sigma_{j}}\left\|\eta_{j}\right\|^{2}<\infty \text {. }
$$

A morphism $\Psi: \Phi \rightarrow \Phi^{\prime}$ consists of a bijection $f: I \stackrel{\sim}{\rightarrow} I^{\prime}$ which satisfies $\sigma_{j}=$ $\sigma_{f(j)}$ and a family of morphisms of Frobenius algebras $\psi_{j}: F_{j} \rightarrow F_{f(j)}^{\prime}$ (which are automatically invertible [Koc, Lem.2.4.5]). We will write $\Psi=\left(f,\left\{\psi_{j}\right\}_{j \in I}\right)$.

Let $\Phi \in \dagger-\mathcal{F} r o b^{\mathrm{F}}$ with the notation from above. For $j \in I$ we turn the Frobenius algebra $F_{j}$ into an RFA by multiplying its structure morphisms by $e^{-a \sigma_{j}}$. Using Proposition 2.16, we get an RFA structure on $\bigoplus_{j \in I} F_{j}$. The next theorem shows that the resulting functor is an equivalence.

Theorem 2.19. There is an equivalence of categories $\dagger-\mathcal{F} r o b^{\mathrm{F}} \rightarrow \dagger-\mathcal{R} \mathcal{F}$ rob given by $\Phi \mapsto \bigoplus_{j \in I} F_{j}{ }^{4}$

Proof. We define the inverse functor. Let $F \in \dagger-\mathcal{R} \mathcal{F}$ rob and fix $a \in \mathbb{R}_{>0}$. Then $P_{a}$ is self-adjoint and therefore can be diagonalised. Let $\operatorname{sp}_{\mathrm{pt}}\left(P_{a}\right)$ denote the point spectrum 5 of $P_{a}$. Furthermore, by Lemma $2.9 P_{a}$ is of trace class, and hence compact. Thus it has at most countably many eigenvalues and the eigenspaces with non-zero eigenvalues are finite-dimensional. Let

$$
F=\bigoplus_{\alpha \in \operatorname{sp}_{\mathrm{pt}}\left(P_{a}\right)} F_{\alpha}
$$

be the corresponding eigenspace decomposition of $P_{a}$.

Claim: The eigenvalue $\alpha$ of $P_{a}$ on $F_{\alpha}$ is of the form $e^{-a \sigma_{\alpha}}$ for some $\sigma_{\alpha} \in \mathbb{R}$. In particular 0 is not an eigenvalue.

To show this, first assume that $c(a):=\alpha \neq 0$, so that $F_{\alpha}$ is finite-dimensional, and simultaneously diagonalise $P_{a}, P_{b}$ and $P_{a+b}$ on $F_{\alpha}$. Then on a subspace where all three operators are constant with values $c(a), c(b)$ and $c(a+b)$ one has that $c(a) c(b)=c(a+b)$. Furthermore $a \mapsto c(a)$ is a continuous function $\mathbb{R}_{\geq 0} \rightarrow \mathbb{R}$ and $c(0)=1$ since $a \mapsto P_{a}$ is strongly continuous at every $a \in \mathbb{R}_{\geq 0}$ and $\lim _{a \rightarrow 0} P_{a}=\mathrm{id}_{F}$. So the unique solution to the above functional equation is $c(\bar{a})=e^{-a \sigma_{\alpha}}$ for some $\sigma_{\alpha} \in \mathbb{R}$.

Finally let us assume that $\alpha=0$. Clearly, $\operatorname{ker}\left(P_{a}\right) \subseteq \operatorname{ker}\left(P_{a+b}\right)$ for every $b \in \mathbb{R}_{\geq 0}$. Since $P_{a}$ is self-adjoint, we have for $v \in F_{0}$ that $0=P_{a}(v)=P_{a / 2}^{\dagger} \circ P_{a / 2}(v)$. But then $P_{a / 2}(v)=0$ and similarly, for every $n \in \mathbb{Z}_{\geq 0}$ we have that $P_{a / 2^{n}}(v)=0$. Altogether we have that $F_{0}=\operatorname{ker}\left(P_{a}\right)=\operatorname{ker}\left(P_{b}\right)$ for every $b \in \mathbb{R}_{\geq 0}$. So $\lim _{a \rightarrow 0} P_{a}=\operatorname{id}_{F} \operatorname{implies}$ that $F_{0}=\{0\}$.

\footnotetext{
${ }^{4}$ We would like to thank André Henriques for explaining to us this decomposition of $\dagger$-RFAs, or rather the corresponding decomposition of Hermitian area-dependent QFTs via Corollary 3.7.

5 The point spectrum of a bounded operator is the set of eigenvalues. Every compact operator on an infinitedimensional Hilbert space has 0 in its spectrum, but it need not be an eigenvalue.
} 
Claim: The eigenspaces are $\dagger$-Frobenius algebras by restricting and projecting the structure maps of $F$.

To show this, first confirm that the structure maps do not mix eigenspaces of $P_{a}$, because $P_{a}$ commutes with them. Then checking $\dagger$-RFA relations is straightforward and these are $\dagger$-Frobenius algebras, cf. Proposition 2.14.

Claim: The convergence conditions in (2.27) are satisfied by the above obtained family of $\dagger$-Frobenius algebras $F_{\alpha}$ and real numbers $\sigma_{\alpha}$.

This can be shown directly by computing the norm of the structure maps.

Showing that the two functors give an equivalence of categories is now straightforward.

Corollary 2.20. Let $A \in \mathcal{H}$ ilb be a $\dagger$-RFA. Then $P_{a}$ is mono and epi.

Proof. From the proof of Theorem 2.19 we see that $P_{a}$ is mono. Since $P_{a}$ is self-adjoint we get that $P_{a}$ is epi.

Lemma 2.21. 1. Every $\dagger$-Frobenius algebra in Hilb is separable, hence semisimple.

2. Every $\dagger-R F A$ is separable.

\section{Proof. Part 1:}

Let $F$ denote a $\dagger$-Frobenius algebra in $\mathcal{H i l b}$ and let $\xi:=\mu \circ \Delta=\Delta^{*} \circ \Delta$, which is an $F-F$-bimodule morphism and an $F-F$-bicomodule morphism. It is a self-adjoint operator, so it can be diagonalised and $F$ decomposes into Hilbert spaces as

$$
F=\bigoplus_{\alpha \in \operatorname{sp}(\xi)} F_{\alpha}
$$

where $F_{\alpha}$ is the eigenspace of $\xi$ with eigenvalue $\alpha$.

Now we show that (2.29) is a direct sum of Frobenius algebras. Let $\alpha \neq \beta$ and take $a \in F_{\alpha}, b \in F_{\beta}$. We have

$$
\begin{aligned}
\xi(a b) & =a \xi(b)=\beta a b \\
& =\xi(a) b=\alpha a b
\end{aligned}
$$

since $\xi$ is a bimodule morphism. Then (2.30) shows that $a b=0$, so (2.29) is a decomposition as algebras.

Similarly one shows that (2.29) is a decomposition as coalgebras. We have for every $a \in F_{\alpha}$, using Sweedler notation:

$$
\begin{aligned}
\Delta(\xi(a)) & =\xi\left(a_{(1)}\right) \otimes a_{(2)}=a_{(1)} \otimes \xi\left(a_{(2)}\right) \\
& =\alpha \Delta(a)=\alpha a_{(1)} \otimes a_{(2)},
\end{aligned}
$$

which shows that the comultiplication restricted to $F_{\alpha}$ lands in $F_{\alpha} \otimes F_{\alpha}$.

We now show that 0 is not in the spectrum. Let us assume otherwise. Then $F_{0}$ is a Frobenius algebra. We have $\xi(x)=\Delta^{*} \circ \Delta(x)=0$ for every $x \in F_{0}$, and so also $\Delta(x)=0$, which is a contradiction to counitality. Therefore 0 is not in the spectrum of $\xi$, i.e. $\xi$ is injective.

Now the only thing left to show is that each summand $F_{\alpha}$ is semisimple. Take $\Delta(1)$. $\alpha^{-1}$ projected on $F_{\alpha} \otimes F_{\alpha}$ and denote it by $e^{\alpha}$. This is a separability idempotent for the algebra $F_{\alpha}$, hence $F_{\alpha}$ is separable, hence semisimple (see e.g. [Pie, Ch. 10.4]). Part 2: 
By Theorem 2.19 we can decompose a $\dagger$-RFA $A=\bigoplus_{\sigma \in I} A_{\sigma}$ into $\dagger$-Frobenius algebras and by the above proof we can decompose the $A_{\sigma}$ summands into finite dimensional separable algebras $A_{\sigma}=\bigoplus_{\alpha} A_{\sigma, \alpha}$ with separability idempotents $e^{\sigma, \alpha}$. We have

$$
\begin{aligned}
\left\|e^{\sigma, \alpha}\right\|^{2} & =\left\|\alpha^{-1} \cdot \Delta^{\sigma, \alpha}(1)\right\|^{2} \stackrel{\left(C^{*}\right)}{=}\left\|\alpha^{-2} \cdot \varepsilon^{\sigma, \alpha} \circ \mu^{\sigma, \alpha} \circ \Delta^{\sigma, \alpha}(1)\right\| \\
& =\left\|\alpha^{-1} \cdot \varepsilon^{\sigma, \alpha}(1)\right\| \stackrel{\left(C^{*}\right)}{=} \alpha^{-1} \cdot\left\|\eta^{\sigma, \alpha}\right\|^{2},
\end{aligned}
$$

where $\left(C^{*}\right)$ refers to the fact that $\left\|X^{\dagger} X\right\|=\|X\|^{2}$ and in the middle step we used that $A_{\sigma, \alpha}$ is the eigenspace of $\xi^{\sigma}=\mu^{\sigma} \circ \Delta^{\sigma}$ with eigenvalue $\alpha$. Furthermore we have that

$$
\begin{aligned}
1 & =\left\|\operatorname{id}_{A_{\sigma, \alpha}}\right\|^{2}=\left\|\mu^{\sigma, \alpha} \circ\left(\eta^{\sigma, \alpha} \otimes \mathrm{id}\right)\right\|^{2} \\
& \leq\left\|\mu^{\sigma, \alpha}\right\|^{2} \cdot\left\|\eta^{\sigma, \alpha}\right\|^{2} \stackrel{\left(C^{*}\right)}{=}\left\|\mu^{\sigma, \alpha} \circ \Delta^{\sigma, \alpha}\right\| \cdot\left\|\eta^{\sigma, \alpha}\right\|^{2}=\alpha \cdot\left\|\eta^{\sigma, \alpha}\right\|^{2} .
\end{aligned}
$$

We claim that

$$
e_{a}:=\sum_{\substack{\sigma \in I \\ \alpha \in \operatorname{sp}\left(\xi^{\sigma}\right)}} e^{-a \sigma} e^{\sigma, \alpha} \in A \otimes A,
$$

which is then clearly a separability idempotent for the RFA $A$. To check (2.34) we compute

$$
\begin{gathered}
\left\|e_{a}\right\|^{2}=\sum_{\substack{\sigma \in I \\
\alpha \in \operatorname{sp}\left(\xi^{\sigma}\right)}} e^{-2 a \sigma}\left\|e^{\sigma, \alpha}\right\|^{2}=\sum_{\substack{\sigma \in I \\
\alpha \in \operatorname{sp}\left(\xi^{\sigma}\right)}} e^{-2 a \sigma} \alpha^{-1}\left\|\eta^{\sigma, \alpha}\right\|^{2} \\
\stackrel{(2.33)}{\leq} \sum_{\substack{\sigma \in I \\
\alpha \in \operatorname{sp}\left(\xi^{\sigma}\right)}} e^{-2 a \sigma}\left\|\eta^{\sigma, \alpha}\right\|^{4} \leq\left\|\eta_{a / 2}\right\|^{4}<\infty .
\end{gathered}
$$

Let $\epsilon \in \mathbb{C} \backslash\{0\}, \sigma \in \mathbb{R}$ and let $\mathbb{C}_{\epsilon, \sigma}$ denote the one-dimensional $\dagger$-RFA structure on $\mathbb{C}$ given by

$\varepsilon_{a}(1)=e^{-a \sigma} \epsilon, \quad \Delta_{a}(1)=\frac{e^{-a \sigma}}{\epsilon} 1 \otimes 1, \quad \eta_{a}(1)=e^{-a \sigma} \epsilon^{*} 1, \quad \mu_{a}(1 \otimes 1)=\frac{e^{-a \sigma}}{\epsilon^{*}} 1$.

Let $C \in \mathcal{H}$ ilb be a one-dimensional $\dagger$-RFA and $c \in C$ with $\|c\|=1$. Then by Proposition 2.14, $\varepsilon_{a}=\varepsilon_{0} \circ P_{a}$ with $P_{a}=e^{-a \sigma} \operatorname{id}_{C}$ for $\sigma \in \mathbb{R}$. Set $\epsilon:=\varepsilon_{0}(c) \in \mathbb{C}$. Then

$$
\begin{aligned}
C & \rightarrow \mathbb{C}_{\epsilon, \sigma} \\
c & \mapsto 1
\end{aligned}
$$

is an isometric isomorphism of RFAs.

Corollary 2.22. Let $C$ be a commutative $\dagger$-RFA. Then there is a family of numbers $\left(\epsilon_{j}, \sigma_{j}\right)_{j \in I}$, where $\epsilon_{j} \in \mathbb{C}$ and $\sigma_{j} \in \mathbb{R}$, satisfying

$$
\sup _{j \in I}\left\{e^{-a \sigma_{j}}\left|\epsilon_{j}\right|^{-1}\right\}<\infty \text { and } \sum_{j \in I} e^{-2 a \sigma_{j}}\left|\epsilon_{j}\right|^{2}<\infty
$$

for every $a \in \mathbb{R}_{>0}$ such that $C \cong \bigoplus_{j \in I} \mathbb{C}_{\epsilon_{j}, \sigma_{j}}$ as RFAs. 
Proof. By Theorem 2.19 and Lemma 2.21, $C$ is a direct sum of semisimple algebras. By the Wedderburn-Artin theorem every semisimple commutative algebra is a direct sum of one-dimensional algebras. Using the isomorphism (2.37) we get the above family of numbers. The finiteness conditions come from (2.27).

Lemma 2.23. Let $\varphi: \mathbb{C}_{\epsilon, \sigma} \rightarrow \mathbb{C}_{\epsilon^{\prime}, \sigma^{\prime}}$ be a morphism of RFAs. Then $\varphi(1)=\epsilon / \epsilon^{\prime} \in U(1)$ and $\sigma=\sigma^{\prime}$.

Proof. From $\varphi \circ \eta_{a}=\eta_{a}^{\prime}$ one has that for every $a \in \mathbb{R}_{\geq 0}, \varphi(1) \epsilon^{*} e^{-a \sigma}=\left(\epsilon^{\prime}\right)^{*} e^{-a \sigma^{\prime}}$. Since $\epsilon \neq 0, \epsilon^{\prime} \neq 0$ and $\varphi(1) \neq 0$, one must have $\sigma=\sigma^{\prime}$ and hence $\varphi(1) \epsilon^{*}=\left(\epsilon^{\prime}\right)^{*}$. One similarly obtains from $\varepsilon_{a}^{\prime} \circ \varphi=\varepsilon_{a}$ that $\epsilon^{\prime} \varphi(1)=\epsilon$. Combining these we get that $|\varphi(1)|=1$ and that $\varphi(1)=\epsilon / \epsilon^{\prime}$.

Proposition 2.24. Every morphism of commutative $\dagger-R F A s$ is unitary, in particular the category of commutative $\dagger$-RFAs is a groupoid.

Proof. Let $\phi: C \rightarrow C^{\prime}$ be a morphism of commutative $\dagger$-RFAs. By Corollary 2.22 we assume that $C=\bigoplus_{j \in I} \mathbb{C}_{\epsilon_{j}, \sigma_{j}}$ and $C^{\prime}=\bigoplus_{j \in I^{\prime}} \mathbb{C}_{\epsilon_{j}^{\prime}, \sigma_{j}^{\prime}}$. By a similar argument as in the proof of Lemma 2.23, we see that $\phi$ does not mix the $\mathbb{C}_{\epsilon_{j}, \sigma_{j}}$ 's with different $\sigma$ 's. Let $C_{\sigma}:=\bigoplus_{\substack{j \in I \\ \sigma_{j}=\sigma}} \mathbb{C}_{\epsilon_{j}, \sigma_{j}}$ and define $C_{\sigma}^{\prime}$ similarly. These are both finite-dimensional, since these are eigenspaces of the $P_{a}$ 's with eigenvalue $e^{-a \sigma}$. Let $\varphi:=\left.\phi\right|_{C_{\sigma}}$. Then $\varphi$ is a morphism of finite-dimensional RFAs so it is a bijection as in particular $\varphi$ is a morphism of Frobenius algebras, cf. the proof of Proposition 2.14. Let $n_{\sigma}:=\operatorname{dim}\left(C_{\sigma}\right)$ and let us write $g_{j}=1\left(j=1, \ldots, n_{\sigma}\right)$ for the generator of $\mathbb{C}_{\epsilon_{j}, \sigma_{j}}$ in $C_{\sigma}$ and $g_{j}^{\prime}=1$ $\left(j=1, \ldots, n_{\sigma}\right)$ for the generator of $\mathbb{C}_{\epsilon_{j}^{\prime}, \sigma_{j}^{\prime}}$ in $C_{\sigma}^{\prime}$ and write $\varphi\left(g_{j}\right)=\sum_{k=1}^{n_{\sigma}} \varphi^{j k} g_{k}^{\prime}$.

From the equation $\varphi \circ \mu=\mu^{\prime} \circ(\varphi \otimes \varphi)$ one has for every $j, k, l$ that

$$
\delta_{j k}\left(\epsilon_{j}^{*}\right)^{-1} \varphi^{j l}=\varphi^{j l} \varphi^{k l}\left(\left(\epsilon_{l}^{\prime}\right)^{*}\right)^{-1} .
$$

- If $j \neq k$ then $\varphi^{j l} \varphi^{k l}=0$ for every such $k$ and for every $l$. This means that in the matrix $\varphi^{j l}$ in every row there might be at most one nonzero element. Since $\varphi$ is bijective there is also at least one nonzero element in every row in the latter matrix and the same holds for every column. We conclude that the matrix of $\varphi$ is the product of a permutation matrix $\rho$ and a diagonal matrix $D$.

- If $j=k$ and if $\varphi^{j l} \neq 0$ then $\varphi^{j l}=\left(\epsilon_{l}^{\prime} / \epsilon_{j}\right)^{*}$, which give the nonzero elements of the diagonal matrix.

Now $\rho^{-1} \circ \varphi$ restricts to RFA morphisms of the one-dimensional components, hence by Lemma 2.23 the diagonal matrix $D$ is unitary. Therefore $\varphi$ is unitary, $\phi$ is the direct sum of unitary matrices so $\phi$ is unitary and in particular invertible.

\subsection{Examples of RFAs.}

\section{Commutative RFAs}

1. Let $\left(\epsilon_{k}, \sigma_{k}\right)_{k \in I}$ be a countable family of pairs of complex numbers such that for all $a>0$

$$
\sup _{k \in I}\left|\epsilon_{k} e^{-a \sigma_{k}}\right|<\infty \text { and } \sum_{k \in I}\left|\frac{e^{-a \sigma_{k}}}{\epsilon_{k}}\right|^{2}<\infty \text {. }
$$


Then $A_{\epsilon, \sigma}:=\bigoplus_{k \in I} \mathbb{C} f_{k}$, the Hilbert space generated by orthonormal vectors $f_{k}$, becomes an RFA by Proposition 2.16 via

$$
\begin{aligned}
\mu_{a}\left(f_{k} \otimes f_{j}\right):=\delta_{k, j} \epsilon_{k} f_{k} e^{-a \sigma_{k}}, & \eta_{a}(1):=\sum_{k \in I} \frac{f_{k}}{\epsilon_{k}} e^{-a \sigma_{k}}, \\
\Delta_{a}\left(f_{k}\right):=\frac{f_{k} \otimes f_{k}}{\epsilon_{k}} e^{-a \sigma_{k}}, & \varepsilon_{a}\left(f_{k}\right):=\epsilon_{k} e^{-a \sigma_{k}}
\end{aligned}
$$

This RFA is strongly separable (with $\tau_{a}=\eta_{a}$ ) and commutative. Furthermore this RFA is hermitian if and only if $\epsilon_{k} \in U(1)$ and $\sigma_{k} \in \mathbb{R}$ for every $k \in I$.

2. Let $I:=\mathbb{Z}_{>0}$ and consider the one-dimensional Hilbert spaces $\mathbb{C} f_{k}$ and $\mathbb{C} g_{k}$ with $\left\|f_{k}\right\|^{2}=k^{2}$ and $\left\|g_{k}\right\|^{2}=k^{-1}$. Let $F:=\bigoplus_{k=1}^{\infty} \mathbb{C} f_{k}$ and $G:=\bigoplus_{k=1}^{\infty} \mathbb{C} g_{k}$ be the Hilbert space direct sums, so that

$$
\left\langle f_{k}, f_{j}\right\rangle_{F}=\delta_{k, j} k^{2} \text { and }\left\langle g_{k}, g_{j}\right\rangle_{G}=\delta_{k, j} k^{-1} \text {. }
$$

Define the maps

$$
\begin{aligned}
\mu_{a}^{F}\left(f_{k} \otimes f_{j}\right) & :=\delta_{k, j} e^{-a k^{2}} f_{k}, \quad \eta_{a}^{F}(1):=\sum_{k=1}^{\infty} e^{-a k^{2}} f_{k}, \\
\Delta_{a}^{F}\left(f_{k}\right) & :=e^{-a k^{2}} f_{k} \otimes f_{k}, \quad \varepsilon_{a}^{F}\left(f_{k}\right):=e^{-a k^{2}},
\end{aligned}
$$

and similarly for $G$ by changing $f_{k}$ to $g_{k}$. These formulas define strongly separable (with $\left.\tau_{a}=\eta_{a}\right)$ commutative RFAs by the previous example with $\left(\epsilon_{k}, \sigma_{k}\right)=\left(k^{-1}, k^{2}\right)$ for $F$ and with $\left(\epsilon_{k}, \sigma_{k}\right)=\left(k, k^{2}\right)$ for $G$. Note that $\lim _{a \rightarrow 0} \mu_{a}^{F}$ exists and has norm 1, but $\lim _{a \rightarrow 0} \mu_{a}^{G}$ does not: the set $\left\{\left\|\mu_{0}^{G}\left(g_{k} \otimes g_{k}\right)\right\| /\left\|g_{k} \otimes g_{k}\right\|=k \mid k \in \mathbb{Z}_{>0}\right\}$ is not bounded.

Define the morphism of RFAs $\psi: F \rightarrow G$ as $\psi\left(f_{k}\right)=g_{k}$. It is an operator with $\|\psi\|=1$ and is mono and epi, but it does not have a bounded inverse, as the set $\left\{\left\|\psi^{-1}\left(g_{k}\right)\right\| /\left\|g_{k}\right\|=k^{2} \mid k \in \mathbb{Z}_{>0}\right\}$ is not bounded. The example also shows that RFA morphisms which are mono and epi need not preserve the existence of zero-area limits. Isomorphisms, on the other hand, being continuous with continuous inverse, do preserve the existence of limits.

These two RFAs are not $\dagger$-RFAs, as one can easily confirm that the summands $\mathbb{C} f_{k}$ and $\mathbb{C} g_{k}$ for $k>1$ are not $\dagger$-RFAs. We compute e.g. for $\mathbb{C} f_{k}$ that

$$
\left\langle f_{k}, \mu_{a}\left(f_{k} \otimes f_{k}\right)\right\rangle=e^{-a k^{2}} k^{2} \quad \text { and } \quad\left\langle\Delta_{a}\left(f_{k}\right), f_{k} \otimes f_{k}\right\rangle=e^{-a k^{2}} k^{4}
$$

so clearly, if $k>1$ then these are not equal and hence $\mu_{a}^{\dagger} \neq \Delta_{a}$.

Remark 2.25. In some cases none of the structure maps of a commutative Hermitian RFA admit an $a \rightarrow 0$ limit. A concrete example can be given as follows. Fix $1 / 2>\delta>0$. Then the family of numbers $\left(n^{1 / 2+\delta}, n\right)_{n \in \mathbb{Z}_{>0}}$ satisfies (2.38) and the structure maps $\mu_{a}$, $\Delta_{a}, \eta_{a}, \varepsilon_{a}$ of the corresponding commutative $\dagger$-RFA from Corollary 2.22 do not have an $a \rightarrow 0$ limit. 


\section{Hermitian RFAs from compact Lie groups}

3. Consider $L^{2}(G)$, the Hilbert space of square integrable functions on a compact semisimple Lie group $G$ with the following morphisms:

$$
\begin{aligned}
\eta_{a}(1) & :=\sum_{V \in \hat{G}} e^{-a \sigma_{V}} \operatorname{dim}(V) \chi_{V}, \quad \mu(F)(x):=\int_{G} F\left(y, y^{-1} x\right) d y, \\
P_{a}(f) & :=\mu\left(\eta_{a}(1) \otimes f\right), \quad \mu_{a}:=P_{a} \circ \mu, \\
\varepsilon_{a}(f) & :=\int_{G} \eta_{a}(1)(x) f\left(x^{-1}\right) d x, \quad \Delta(f)(x, y):=f(x y), \quad \Delta_{a}:=\Delta \circ P_{a},
\end{aligned}
$$

where $f \in L^{2}(G), F \in L^{2}(G \times G) \cong L^{2}(G) \otimes L^{2}(G), \hat{G}$ is a set of representatives of isomorphism classes of finite-dimensional simple unitary $G$-modules, $\sigma_{V}$ is the value of the Casimir operator of the Lie algebra of $G$ in the simple module $V, \chi_{V}$ is the character of $V$, and $\int_{G}$ denotes the Haar integral on $G$. These formulas define a strongly separable symmetric RFA (with $\tau_{a}=\eta_{a}$ ), for details see Sect. 5.1.

4. The centre of the previous RFA is $C l^{2}(G)$, the Hilbert space of square integrable class functions on $G$, with multiplication, unit and counit given by the same formulas, but with the following coproduct:

$$
\Delta_{a}(f)=\sum_{V \in \hat{G}} e^{-a \sigma_{V}}(\operatorname{dim}(V))^{-1} \chi_{V} \otimes \chi_{V} f_{V},
$$

where $f=\sum_{V \in \hat{G}} f_{V} \chi_{V} \in C l^{2}(G)$. This is a strongly separable commutative RFA (with $\tau_{a}(1)=\sum_{V \in \hat{G}} e^{-a \sigma_{V}}(\operatorname{dim}(V))^{-1} \chi_{V}$ and $\tau_{a}^{-1}(1)=\sum_{V \in \hat{G}} e^{-a \sigma_{V}}(\operatorname{dim}(V))^{3}$ $\left.\chi_{V}\right)$. For more details see Sect. 5.1.

\section{Area-Dependent QFTs as Functors}

In this section we define the symmetric monoidal category of two-dimensional bordisms with area. Using this, area-dependent QFTs are defined as symmetric monoidal functors from such bordisms to the category of Hilbert spaces $\mathcal{H} i l b$. We classify such functors in terms of commutative regularised Frobenius algebras, mirroring the result for twodimensional TQFTs.

Below, by manifold we will always mean an oriented smooth manifold.

3.1. Bordisms with area and aQFTs. Recall the definition of the category of 2-dimensional oriented bordisms Bord $_{2}$ [Koc, Car]: The objects are closed 1-dimensional manifolds and morphisms are diffeomorphism classes of compact surfaces with boundary parametrisations that identify the boundary with the source and target 1-manifolds. The category of bordisms with area $\mathcal{B o r d}_{2}^{\text {area }}$ has the same objects as $\mathcal{B}$ ord $d_{2}$ and the morphism are pairs

$$
\left(\Sigma, \mathcal{A}: \pi_{0}(\Sigma) \rightarrow \mathbb{R}_{\geq 0}\right)
$$

where $\Sigma$ is a morphism in $\mathcal{B o r d}_{2}$ and $\mathcal{A}$ assigns an area to each of its connected components, which is additive under composition. We allow $\mathcal{A}$ to take 0 value only on cylinders. 
Here, by cylinder we mean bordisms $C: S \rightarrow S^{\prime}$, where $S \cong S^{\prime}$ as 1-manifolds, and where $C$ is diffeomorphic to $\mathbb{S}^{1} \times[0,1]$ as a 2 -manifold with boundary. This implies that Bord $_{2}^{\text {area }}$ has identity morphisms, and that objects given by diffeomorphic 1-manifolds are isomorphic in $\mathcal{B o r d}_{2}^{\text {area }}$. Both $\mathcal{B o r d}_{2}$ and $\mathcal{B o r d}_{2}^{\text {area }}$ are symmetric monoidal with the disjoint union as tensor product.

We equip the hom-sets of $\mathcal{B o r d}_{2}^{\text {area }}$ with a topology as follows. Fix a bordism $\Sigma$ : $S \rightarrow T$ in $\mathcal{B o r d}_{2}$. Define the subset $U_{\Sigma} \subset \mathcal{B o r d}_{2}^{\text {area }}(S, T)$ as

$$
U_{\Sigma}:=F^{-1}(\Sigma)=\left\{(\Sigma, \mathcal{A}) \mid \mathcal{A}: \pi_{0}(\Sigma) \rightarrow \mathbb{R}_{\geq 0}\right\} \cong\left(\mathbb{R}_{>0}\right)^{N_{n}} \times\left(\mathbb{R}_{\geq 0}\right)^{N_{c}}
$$

where $N_{c}$ is the number of connected components of $\Sigma$ equivalent to a cylinder over a connected 1-manifold and $N_{n}=\left|\pi_{0}(\Sigma)\right|-N_{c}$. The topology on $U_{\Sigma}$ is that of $\left(\mathbb{R}_{>0}\right)^{N_{n}} \times$ $\left(\mathbb{R}_{\geq 0}\right)^{N_{c}}$. We define the topology on $\operatorname{Bord}_{2}^{\text {area }}(S, T)$ to be the disjoint union topology of the sets $U_{\Sigma}$. After these preparations we can define:

Definition 3.1. An area-dependent quantum field theory, or aQFT for short, is a symmetric monoidal functor $\mathcal{Z}: \mathcal{B o r d}_{2}^{\text {area }} \rightarrow \mathcal{H i l b}$, such that for every $S, T \in \mathcal{B o r d}_{2}^{\text {area }}$ the map

$$
\begin{aligned}
\mathcal{Z}_{S, T}: \operatorname{Bord}_{2}^{\text {area }}(S, T) & \rightarrow \mathcal{B}(\mathcal{Z}(S), \mathcal{Z}(T)) \\
(\Sigma, \mathcal{A}) & \mapsto \mathcal{Z}(\Sigma, \mathcal{A})
\end{aligned}
$$

is continuous.

Remark 3.2. Bord $_{2}^{\text {area }}$ is enriched in topological spaces $\mathcal{T}$ op, thus one could try to define area-dependent theories to be $\mathcal{T}$ op-enriched symmetric monoidal functors $\mathcal{B o r d}_{2}^{\text {area }} \rightarrow$ $\mathcal{H} i l b$ without the explicit mention of continuity in the area. However this would be too restrictive: The category $\mathcal{H i l b}$ with strong operator topology is not $\mathcal{T}$ op-enriched [KR, Sec. 2.6]. On the other hand, Hilb with norm topology is $\mathcal{T} o p$-enriched, but it leads to the problem already encountered in Corollary 2.10: The existence of zero area limits of cylinders implies that all Hilbert spaces $\mathcal{Z}(S)$ are finite-dimensional.

The following lemma shows that it is enough to require continuity in the area to hold for cylinders over $\mathbb{S}^{1}$. The proof is similar to the proof of Lemma 2.5 and we omit it.

Lemma 3.3. Let $\mathcal{Z}:$ Bord $_{2}^{\text {area }} \rightarrow$ Hilb be a symmetric monoidal functor and let $\left(\mathbb{S}^{1} \times[0,1], a\right)$ denote a cylinder with area $a$. If the assignment

$$
\begin{aligned}
\mathbb{R}_{\geq 0} & \rightarrow \mathcal{B}\left(\mathcal{Z}\left(\mathbb{S}^{1}\right), \mathcal{Z}\left(\mathbb{S}^{1}\right)\right) \\
a & \mapsto \mathcal{Z}\left(\mathbb{S}^{1} \times[0,1], a\right),
\end{aligned}
$$

is continuous, then $\mathcal{Z}$ is an aQFT.

Similarly to RFAs, aQFTs form a symmetric monoidal category $a Q F T$.

The categories $\mathcal{B o r d}_{2}$ and $\mathcal{B o r d}_{2}^{\text {area }}$ are $\dagger$-categories, where $(-)^{\dagger}$ is the identity on objects. On morphisms it flips the orientation of a surfaces and switches its in- and outgoing boundary components, while keeping its area. Following the terminology of [Tur, Sec.5.2] we define: 
Definition 3.4. We call an aQFT $\mathcal{Z}: \mathcal{B o r d}_{2}^{\text {area }} \rightarrow \mathcal{H i l b}$ Hermitian, if the diagram

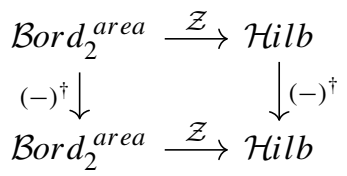

commutes strictly.

3.2. Equivalence of aQFTs and commutative RFAs. Recall that 2-dimensional topological quantum field theories (TQFT) correspond to commutative Frobenius algebras by assigning to a TQFT $\mathcal{Z}$ its value on the circle $\mathcal{Z}\left(\mathbb{S}^{1}\right)$. The structure maps of $\mathcal{Z}\left(\mathbb{S}^{1}\right)$ are given by the value of $\mathcal{Z}$ on the generators of $\mathcal{B o r d} d_{2}$, i.e. on three holed spheres (the two pairs of pants) and on discs (the cup and the cap).

Analogously, if $\mathcal{Z}$ is an aQFT one obtains a commutative RFA structure on $\mathcal{Z}\left(\mathbb{S}^{1}\right)$, the parameter of the families of structure morphisms being the area. We have the following generalisation of [Dij] and [Abr, Thm.3] for 2d TQFTs:

Theorem 3.5. The functor

$$
\begin{aligned}
G: a Q F T & \rightarrow c \mathcal{R} \mathcal{F} r o b \\
\mathcal{Z} & \mapsto \mathcal{Z}\left(\mathbb{S}^{1}\right)
\end{aligned}
$$

is an equivalence of symmetric monoidal categories.

The proof of this theorem is via a generators and relations description of $\mathcal{B o r d}_{2}^{\text {area }}$, essentially the same as in the topological case, see [Sze] for details.

Remark 3.6. If all zero area limits of $\mathcal{Z} \in a Q F T$ exist, then the RFA $\mathcal{Z}\left(\mathbb{S}^{1}\right)$ is finitedimensional. This follows from Theorem 3.5 and Proposition 2.14.

Corollary 3.7. The restriction of the functor $G$ in (3.6) to the category of Hermitian aQFTs gives an equivalence to the category of $\dagger-R F A$ s.

Corollary 2.22 together with Corollary 3.7 shows that a Hermitian aQFT is determined by a countable family of numbers $\left(\epsilon_{i}, \sigma_{i}\right)_{i \in I}$ satisfying convergence conditions given in Corollary 2.22.

\section{State Sum Construction of aQFTs}

The state sum construction of two-dimensional TQFTs (see [BP,FHK] and e.g. [LP, DKR]) has a straightforward generalisation to aQFTs which we investigate in this section. We give an assignment of weights for plaquettes, edges and vertices from a strongly separable symmetric RFA in order to obtain state sum aQFT and describe the connection to the classification of aQFTs in terms of commutative RFAs assigned to the circle. 
(a)

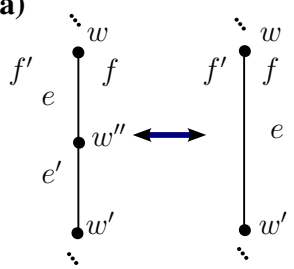

(b)

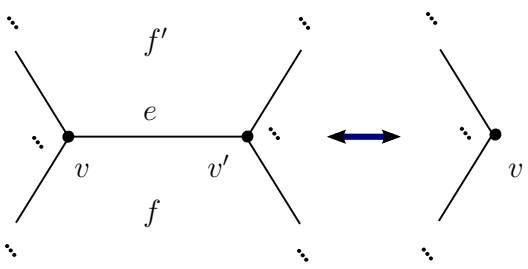

$f^{\prime \prime}$

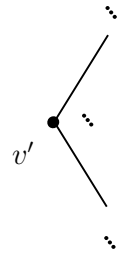

Fig. 2. Elementary moves of PLCW decompositions with area. a shows edges $e, e^{\prime}$ and between faces $f$ and $f^{\prime}$. (The two faces are allowed to be the same.) When we remove the vertex $w^{\prime \prime}$ and the edge $e^{\prime}$, the new area maps should be the same outside the shown region and such that the area of the connected component of the surface does not change. b shows an edge $e$ between two faces $f$ and $f^{\prime}$. When we remove the edge $e$ and merge the faces $f$ and $f^{\prime}$ to $f^{\prime \prime}$, the new area maps should again be the same outside the shown region and such that the area of the connected component of the surface does not change

4.1. PLCW decompositions with area. In Sect. 4.2 we will use PLCW decompositions [Kir] to build aQFTs. For a compact surface $\Sigma$ this consists of three sets $\Sigma_{0}, \Sigma_{1}$ and $\Sigma_{2}$ whose elements are subsets of $\Sigma$. Their elements are called vertices, edges and faces. Faces are embeddings of polygons with $n \geq 1$ edges, edges are embeddings of intervals and vertices are just points in $\Sigma$. Faces are glued along edges so that vertices are glued to vertices. For example a PLCW decomposition of a cylinder $\mathbb{S}^{1} \times[0,1]$ could consist of a rectangle with two opposite edges glued together. From this one can obtain a PLCW decomposition of a torus $\mathbb{S}^{1} \times \mathbb{S}^{1}$ by glueing together the other two opposite edges. For more details on PLCW decompositions we refer to [Kir] and for a short summary to [RS, Sec. 2.2].

We are going to need PLCW decompositions of surfaces with area, which we define now. Let $(\Sigma, \mathcal{A})$ be a surface with strictly positive area for each connected component and let $\Sigma_{0}, \Sigma_{1}, \Sigma_{2}$ be a PLCW decomposition of $\Sigma$. Let $\mathcal{A}_{k}: \Sigma_{k} \rightarrow \mathbb{R}_{>0}$ be maps for $k \in\{0,1,2\}$, which assign to vertices, edges and faces an area, such that for every connected component $x \in \pi_{0}(\Sigma)$ the sum of the areas of vertices, edges and faces of $x$ is equal to its area $\mathcal{A}(x)$. A PLCW decomposition of a surface with area $(\Sigma, \mathcal{A})$ consists of a choice of $\Sigma_{k}$ and $\mathcal{A}_{k}$ for $k \in\{0,1,2\}$.

Definition 4.1. An elementary move on a PLCW decomposition of a surface with area is either

- removing or adding a bivalent vertex as in Fig. $2 \mathrm{a}$, or

- removing or adding an edge as in Fig. 2 b.

By [Kir, Thm. 7.4], any two PLCW decompositions can be related by these elementary moves. The elementary moves in Fig. 2 map PLCW decompositions with area to PLCW decompositions with area.

4.2. State sum construction. Let $A \in \mathcal{H}$ ilb be a strongly separable symmetric RFA. Recall that $\tau_{a}^{-1}$ denotes the inverse to window element in the sense of Proposition 2.8. We consider the following families of morphisms

$$
\begin{gathered}
\zeta_{a}:=\mu_{a_{1}} \circ\left(\operatorname{id}_{A} \otimes \tau_{a_{2}}^{-1}\right) \in \mathcal{B}(A, A), \quad \beta_{a}:=\varepsilon_{a_{1}} \circ \mu_{a_{2}} \in \mathcal{B}\left(A^{\otimes 2}, \mathbb{C}\right) \\
\text { and } W_{a}^{n}:=\Delta_{a_{1}}^{(n)} \circ \eta_{a_{2}} \in \mathcal{B}\left(\mathbb{C}, A^{\otimes n}\right)
\end{gathered}
$$


for $a_{1}, a_{2} \in \mathbb{R}_{>0}$ with $a_{1}+a_{2}=a$ and $n \in \mathbb{Z}_{\geq 1}$. Here, $\Delta_{a}^{(n)}$ is the $n$-fold coproduct: $\Delta_{a}^{(1)}=P_{a}, \Delta_{a}^{(2)}=\Delta_{a}, \Delta_{a}^{(n)}=\left(\Delta_{a / n} \otimes \mathrm{id}_{A} \otimes \cdots \otimes \mathrm{id}_{A}\right) \circ \Delta_{a(n-1) / n}^{(n-1)}$. We call $\beta_{a}$ the contraction and $W_{a}^{n}$ the plaquette weights. We will use the following graphical notation for these morphisms:
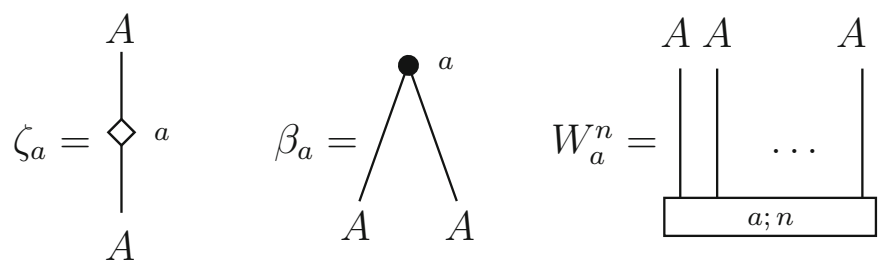

We introduce the family of morphisms $D_{a}: A \rightarrow A$ :

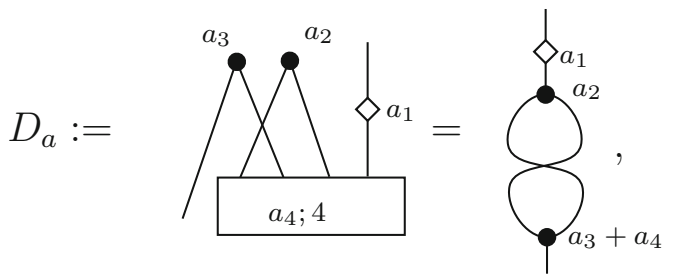

for every $a_{1}, a_{2}, a_{3}, a_{4} \in \mathbb{R}_{>0}$ and $a=\sum_{i=1}^{4} a_{i}$. It follows from the axioms of an RFA that these compositions depend only on the sum of the parameters.

The morphisms in (4.1) and (4.3) satisfy the following conditions for every $a, a_{0}, a_{1}$, $a_{2}, a_{3} \in \mathbb{R}_{>0}$, and for every $n \in \mathbb{Z}_{\geq 1}$ :

1. Cyclic symmetry:

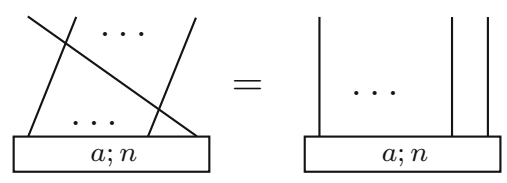

and

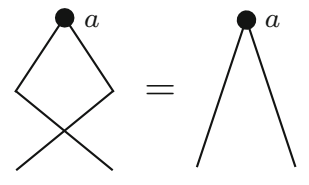

2. Glueing plaquette weights:

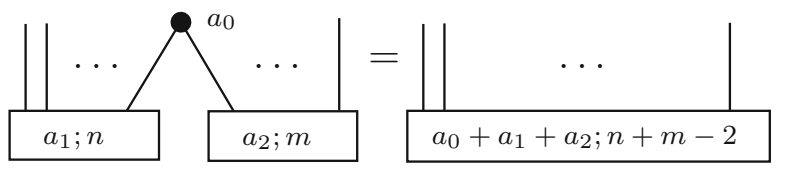

3. Removing a bubble:

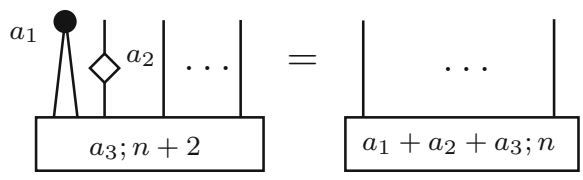


4. Moving $\zeta_{a}$ :

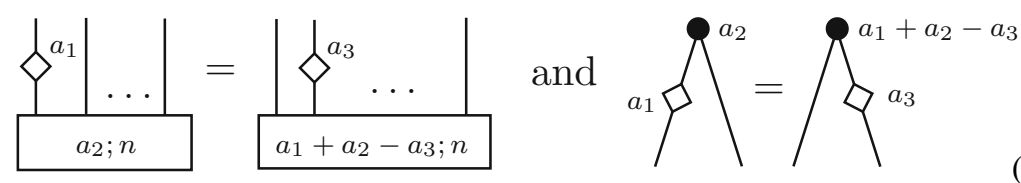

Furthermore we will assume the following:

(L) The limit $\lim _{a \rightarrow 0} D_{a}$ exists.

We note that this assumption is quite natural, as it holds for all examples of the form given in Proposition 2.16. In this case, the limit is the sum of finite rank projections. Furthermore, from Lemma 2.21 Part 2 and Remark 2.17 we get the following lemma:

Lemma 4.2. A hermitian symmetric RFA is strongly separable and satisfies assumption (L).

The following lemma is a direct generalisation of [LP, Prop. 2.20].

Lemma 4.3. Let A be strongly separable symmetric RFA satisfying Assumption ( $L)$. Then $D_{a} \circ D_{b}=D_{a+b}$ for every $a, b \in \mathbb{R}_{>0}$ and the image of the idempotent $D_{0}:=\lim _{a \rightarrow 0} D_{a}$ is the centre $Z(A)$ of $A$. It is an RFA with the restricted structure maps of A. Let us write

$$
D_{0}=\left[A \stackrel{\pi_{A}}{\rightarrow} Z(A) \stackrel{\iota_{A}}{\rightarrow} A\right], \quad\left[Z(A) \stackrel{\iota_{A}}{\rightarrow} A \stackrel{\pi_{A}}{\rightarrow} Z(A)\right]=\operatorname{id}_{Z(A)},
$$

for the embedding and projection of $Z(A)$.

In the rest of this section we define a symmetric monoidal functor $\mathcal{Z}_{A}: \mathcal{B o r d}_{2}^{\text {area }} \rightarrow$ $\mathcal{H}$ ilb using the RFA $A$. Let $S \in \mathcal{B o r d}_{2}^{\text {area }}$. Then

$$
\mathcal{Z}_{A}(S):=\bigotimes_{x \in \pi_{0}(S)} Z(A)^{(x)}
$$

where $Z(A)^{(x)}=Z(A)$ and the superscript is used to label the tensor factors.

In the remainder of this section we give the definition of $\mathcal{Z}_{A}$ on morphisms. Let $(\Sigma, \mathcal{A}): S \rightarrow T$ be a bordism with area and let us assume that $(\Sigma, \mathcal{A})$ has no component with zero area. Choose a PLCW decomposition with area $\Sigma_{k}, \mathcal{A}_{k}$ for $k \in\{0,1,2\}$ of the surface with area $(\Sigma, \mathcal{A})$, such that the PLCW decomposition has exactly 1 edge on every boundary component. By this convention $\pi_{0}(S) \sqcup \pi_{0}(T)$ is in bijection with vertices on the boundary and with edges on the boundary.

Let us choose an edge for every face before glueing, which we call marked edge, and let us choose an orientation of every edge. For a face $f \in \Sigma_{2}$ which is an $n_{f}$-gon let us write $(f, k), k=1, \ldots, n_{f}$ for the sides of $f$, where $(f, 1)$ denotes the marked edge of $f$, and the labeling proceeds counter-clockwise with respect to the orientation of $f$. We collect the sides of all faces into a set:

$$
F:=\left\{(f, k) \mid f \in \Sigma_{2}, k=1, \ldots, n_{f}\right\} .
$$

We double the set of edges by considering $\Sigma_{1} \times\{l, r\}$, where "l" and " $r$ " stand for left and right, respectively. Let $E \subset \Sigma_{1} \times\{l, r\}$ be the subset of all $(e, l)$ (resp. $\left.(e, r)\right)$, which have a face attached on the left (resp. right) side, cf. Fig. 3a. Thus for an inner 
(a)

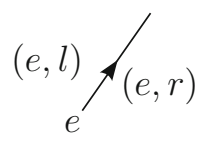

(b)

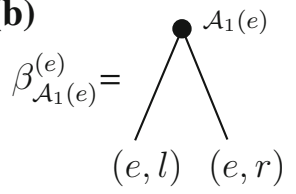

(c)

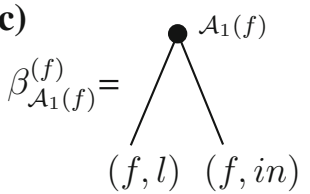

Fig. 3. a Left and right sides $(e, l)$ and $(e, r)$ of an inner edge $e$, determined by the orientation of $\Sigma$ (paper orientation) and of $e$ (arrow). b Convention for connecting tensor factors belonging to edge sides $(e, l)$ and $(e, r)$ of an inner edge $e$ with the tensor factors belonging to the morphism $\beta_{\mathcal{A}_{1}(e)}^{(e)}$. c Conventions for the labels of the tensor factors for an ingoing boundary edge $f$ with $(f, l) \in E$

edge $e \in \Sigma_{1}$ the set $E$ contains both $(e, l)$ and $(e, r)$, but for a boundary edge $e^{\prime} \in \Sigma_{1}$ the set $E$ contains either $\left(e^{\prime}, l\right)$ or $\left(e^{\prime}, r\right)$. By construction of $F$ and $E$ we obtain a bijection

$$
\Phi: F \stackrel{\sim}{\longrightarrow} E, \quad(f, k) \mapsto(e, x),
$$

where $e$ is the $k^{\prime}$ th edge on the boundary of the face $f$ lying on the side $x$ of $e$, counted counter-clockwise from the marked edge of $f$.

For every vertex $v \in \Sigma_{0}$ in the interior of $\Sigma$ or on an ingoing boundary component of $\Sigma$ choose a side of an edge $(e, x) \in E$ for which $v \in \partial(e)$. Let

$$
V: \Sigma_{0} \backslash \pi_{0}(T) \rightarrow E
$$

be the resulting function.

To define $\mathcal{Z}_{A}(\Sigma, \mathcal{A})$ we proceed with the following steps.

1. Let us introduce the tensor products

$$
\begin{array}{ll}
\mathcal{O}_{F}:=\bigotimes_{(f, k) \in F} A^{(f, k)}, & \mathcal{O}_{E}:=\bigotimes_{(e, x) \in E} A^{(e, x)}, \\
\mathcal{O}_{\text {in }}:=\bigotimes_{b \in \pi_{0}(S)} A^{(b, \text { in })}, & \mathcal{O}_{\text {out }}:=\bigotimes_{c \in \pi_{0}(T)} A^{(c, \text { out })} .
\end{array}
$$

Every tensor factor is equal to $A$, but the various superscripts will help us distinguish tensor factors in the source and target objects of the morphisms we define in the remaining steps.

2. Recall that by our conventions there is one edge in each boundary component and that we identified outgoing boundary edges with $\pi_{0}(T)$. Define the morphism

$$
\mathcal{C}:=\bigotimes_{e \in \Sigma_{1} \backslash \pi_{0}(T)} \beta_{\mathcal{A}_{1}(e)}^{(e)}: \mathcal{O}_{\text {in }} \otimes \mathcal{O}_{E} \rightarrow \mathcal{O}_{\text {out }},
$$

where $\beta_{\mathcal{A}_{1}(e)}^{(e)}=\beta_{\mathcal{A}_{1}(e)}$, and where the tensor factors in $\mathcal{O}_{\text {in }} \otimes \mathcal{O}_{E}$ are assigned to those of $\beta_{\mathcal{A}_{1}(e)}$ according to Fig. 3b, c.

3. Define the morphism

$$
\mathcal{Y}:=\prod_{v \in \Sigma_{0} \backslash \pi_{0}(T)} \zeta_{\mathcal{A}_{0}(v)}^{(V(v))} \in \mathcal{B}\left(\mathcal{O}_{E}, \mathcal{O}_{E}\right)
$$

where

$$
\zeta_{a}^{(e, x)}=\mathrm{id} \otimes \cdots \otimes \zeta_{a} \otimes \cdots \otimes \mathrm{id} \in \mathcal{B}\left(\mathcal{O}_{E}, \mathcal{O}_{E}\right),
$$

where $\zeta_{a}$ maps the tensor factor $A^{(e, x)}$ to itself. 
4. Assign to every face $f \in \Sigma_{2}$ obtained from an $n_{f}$-gon the morphism

$$
W_{\mathcal{A}_{2}(f)}^{f}=W_{\mathcal{A}_{2}(f)}^{\left(n_{f}\right)}: \mathbb{C} \rightarrow A_{(f, 1)} \otimes \cdots \otimes A_{\left(f, n_{f}\right)}
$$

and take their tensor product:

$$
\mathcal{F}:=\bigotimes_{f \in \Sigma_{2}}\left(W_{\mathcal{A}_{2}(f)}^{f}\right): \mathbb{C} \rightarrow \mathcal{O}_{F}
$$

5. We will now put the above morphisms together to obtain a morphism $\mathcal{L}: \mathcal{A}_{\text {in }} \rightarrow \mathcal{A}_{\text {out }}$. Denote by $\Pi_{\Phi}$ the permutation of tensor factors induced by $\Phi: F \rightarrow E$,

$$
\Pi_{\Phi}: \mathcal{O}_{F} \rightarrow \mathcal{O}_{E}
$$

Using this, we define

$$
\begin{aligned}
\mathcal{K} & :=\left[\mathbb{C} \stackrel{\mathcal{F}}{\rightarrow} \mathcal{O}_{F} \stackrel{\Pi_{\Phi}}{\rightarrow} \mathcal{O}_{E} \stackrel{\mathcal{Y}}{\rightarrow} \mathcal{O}_{E}\right], \\
\mathcal{L} & :=\left[\mathcal{O}_{\text {in }} \stackrel{\text { id }_{\mathcal{O}_{\text {in }}} \otimes \mathcal{K}}{\longrightarrow} \mathcal{O}_{\text {in }} \otimes \mathcal{O}_{E} \stackrel{\mathcal{C}}{\rightarrow} \mathcal{O}_{\text {out }}\right] .
\end{aligned}
$$

6. Using the embedding and projection maps $\iota_{A}, \pi_{A}$ from (4.8) we construct the morphisms:

$$
\mathcal{E}_{\text {in }}:=\bigotimes_{b \in \pi_{0}(S)} i_{A}^{(b)}: \mathcal{Z}_{A}(S) \rightarrow \mathcal{O}_{\text {in }}, \quad \mathcal{E}_{\text {out }}:=\bigotimes_{c \in \pi_{0}(T)} \pi_{A}^{(c)}: \mathcal{O}_{\text {out }} \rightarrow \mathcal{Z}_{A}(T),
$$

where $\iota_{A}^{(b)}=\iota_{A}: Z(A)^{(b)} \rightarrow A^{(b)}$ and $\pi_{A}^{(b)}=\pi_{A}: A^{(b)} \rightarrow Z(A)^{(b)}$. We have all ingredients to define the action of $\mathcal{Z}_{A}$ on morphisms:

$$
\mathcal{Z}_{A}(\Sigma, \mathcal{A}):=\left[\mathcal{Z}_{A}(S) \stackrel{\mathcal{E}_{\text {in }}}{\rightarrow} \mathcal{O}_{\text {in }} \stackrel{\mathcal{L}}{\rightarrow} \mathcal{O}_{\text {out }} \stackrel{\mathcal{E}_{\text {out }}}{\longrightarrow} \mathcal{Z}_{A}(T)\right] .
$$

Now that we defined $\mathcal{Z}_{A(\mathbb{D})}$ on bordisms with strictly positive area, we turn to the general case. Let $(\Sigma, \mathcal{A}): S \rightarrow T$ be a bordism with area and let $\Sigma_{+}: S_{+} \rightarrow T_{+}$denote the connected component of $(\Sigma, \mathcal{A})$ with strictly positive area. We have that in $\mathcal{B}$ ord ${ }_{2}^{\text {area }}$

$$
(\Sigma, \mathcal{A})=\left(\Sigma_{+}, \mathcal{A}_{+}\right) \sqcup\left(\Sigma \backslash \Sigma_{+}, 0\right),
$$

where $\mathcal{A}_{+}$denotes the restriction of $\mathcal{A}$ to $\pi_{0}\left(\Sigma_{+}\right)$. The bordism with zero area $\left(\Sigma \backslash \Sigma_{+}, 0\right)$ defines a permutation $\kappa: \pi_{0}\left(S \backslash S_{+}\right) \rightarrow \pi_{0}\left(T \backslash T_{+}\right)$. Let $\mathcal{Z}_{A}\left(\Sigma \backslash \Sigma_{+}, 0\right): \mathcal{Z}_{A}\left(S \backslash S_{+}\right) \rightarrow$ $\mathcal{Z}_{A}\left(T \backslash T_{+}\right)$be the induced permutation of tensor factors. We define

$$
\mathcal{Z}_{A}(\Sigma, \mathcal{A}):=\mathcal{Z}_{A}\left(\Sigma \backslash \Sigma_{+}, 0\right) \otimes \mathcal{Z}_{A}\left(\Sigma_{+}, \mathcal{A}_{+}\right)
$$

where $\mathcal{Z}_{A}\left(\Sigma_{+}, \mathcal{A}_{+}\right)$is defined in (4.23).

Theorem 4.4. Let A be a strongly separable symmetric RFA satisfying Assumption ( $L)$.

1. The morphism defined in (4.23) is independent of the choice of the PLCW decomposition with area, the choice of marked edges of faces, the choice of orientation of edges and the assignment $V$. 
2. The state sum construction yields an aQFT $\mathcal{Z}_{A}: \mathcal{B o r d}_{2}^{\text {area }} \rightarrow \mathcal{H i l b}$ whose action on objects and morphisms is given by (4.9) and (4.25), respectively.

3. The commutative RFA corresponding to the $a Q F T \mathcal{Z}_{A}$ is the center of $A$.

\section{Proof. Part 1:}

In order to show independence of the PLCW decomposition with area first notice that all conditions on $A$ depend on the sum of the parameters. This implies that the construction is independent of the distribution of area, i.e. the maps $\mathcal{A}_{k}(k \in\{0,1,2\})$. Checking that the construction is independent of the details of the PLCW decomposition can be done as in the case of TFTs (see e.g. [DKR, Lem. 3.5]), which is due to Conditions 1-4.

\section{Part 2:}

For an in-out cylinder with a single component with area $a$ the morphism $\mathcal{L}$ in from (4.21) is exactly $D_{a}$ from (4.3). Clearly this is continuous in the parameter. When considering an in-out cylinder with several components, the zero area limit of the assigned morphism exists by Assumption (L) and it is clearly a permutation of tensor factors.

Functoriality follows from the fact that the morphisms $D_{a}$ form a semigroup (Lemma 4.3). Monoidality and symmetry directly follow from the construction, continuity in the area follows from Lemma 3.3 , hence $\mathcal{Z}_{A}$ is indeed an aQFT.

Part 3: This directly follows from Lemma 4.3 and from the (4.9).

\section{Example: 2d Yang-Mills Theory}

The state sum construction of 2d Yang-Mills theory has been introduced by [Mig], was further developed for $G=U(N)$ in [Rus], and has been summarised in [Wit]; for a review see [CMR]. There, partition functions and expectation values of Wilson loops were calculated. These references also discuss the relation between the state-sum construction and the Lagrangian-based field theoretic approach to $2 \mathrm{~d}$ Yang-Mills theory.

The proof of convergence of the (Boltzmann) plaquette weights has been shown in a different setting in [App]. In this section we will heavily rely on the representation theory of compact Lie groups, a standard reference is e.g. [Kna].

5.1. Two RFAs from a compact group $G$. Let $G$ be a compact semisimple Lie group and $\int d x$ the Haar integral on $G$ with the normalisation $\int_{G} 1 d x=1$. We denote with $L^{2}(G)$ the Hilbert space of square integrable complex functions on $G$, where the scalar product of $f, g \in L^{2}(G)$ is given by $\langle f, g\rangle:=\int f(x)^{*} g(x) d x$.

Let $\hat{G}$ denote a set of representatives of isomorphism classes of finite-dimensional simple unitary $G$-modules. Then for $V \in \hat{G}$ with inner product $\langle-,-\rangle_{V}$ and an orthonormal basis $\left\{e_{i}^{V}\right\}_{i=1}^{\operatorname{dim}(V)}$ let

$$
\begin{aligned}
f_{i j}^{V}: G & \rightarrow \mathbb{C} \\
g & \mapsto(\operatorname{dim}(V))^{1 / 2}\left\langle e_{i}^{V}, g \cdot e_{j}^{V}\right\rangle_{V}
\end{aligned}
$$

denote a matrix element function and let $M_{V}$ denote the linear span of these. The matrix element functions are orthonormal [Kna, Cor. 4.2]: for $V, W \in \hat{G}, i, j \in\{1, \ldots, \operatorname{dim}(V)\}$ and $k, l \in\{1, \ldots, \operatorname{dim}(W)\}$

$$
\left\langle f_{i j}^{V}, f_{k l}^{W}\right\rangle=\delta_{i k} \delta_{j l} \delta_{V, W}
$$


where $\delta_{V, W}=1$ if $V=W$ and 0 otherwise. The character of $V$ is defined as

$$
\chi_{V}=(\operatorname{dim}(V))^{-1 / 2} \sum_{i=1}^{\operatorname{dim}(V)} f_{i i}^{V} .
$$

The Peter-Weyl theorem provides a complete orthonormal basis of $L^{2}(G)$ in terms of matrix element functions and of the square integrable class functions $C l^{2}(G)$ in terms of characters:

$$
L^{2}(G) \cong \bigoplus_{V \in \hat{G}} M_{V} \text { and } C l^{2}(G) \cong \bigoplus_{V \in \hat{G}} \mathbb{C} \cdot \chi_{V}
$$

as Hilbert space direct sums. Note that $L^{2}(G) \otimes L^{2}(G) \cong L^{2}(G \times G)$ and $C l^{2}(G) \otimes$ $C l^{2}(G) \cong C l^{2}(G \times G)$ isometrically by mapping $f \otimes f^{\prime}$ to the function $\left(g, g^{\prime}\right) \mapsto$ $f(g) f^{\prime}\left(g^{\prime}\right)$. We will often use these isomorphisms without further notice.

In the following we will define a $†$-RFA structure on $L^{2}(G)$ and $C l^{2}(G)$. Let us start with defining the operator

$$
\begin{aligned}
\Delta: L^{2}(G) & \rightarrow L^{2}(G) \otimes L^{2}(G) \\
f & \mapsto \Delta(f)=[(x, y) \mapsto f(x y)],
\end{aligned}
$$

which has norm 1 . Let $\mu:=\Delta^{\dagger}: L^{2}(G) \otimes L^{2}(G) \rightarrow L^{2}(G)$ be its adjoint, which is given by the convolution product. For $F \in L^{2}(G) \otimes L^{2}(G)$

$$
\mu(F)(y)=\int_{G} F\left(x, x^{-1} y\right) d x .
$$

Let $V \in \hat{G}$ and let us denote with $\sigma_{V} \in \mathbb{R}$ the value of the Casimir operator of $G$ in the module $V$. We define the unit to be the heat kernel on $G$ :

$$
\begin{aligned}
\eta_{a}: \mathbb{C} & \rightarrow L^{2}(G) \\
1 & \mapsto \eta_{a}(1)=\sum_{V \in \hat{G}} e^{-a \sigma_{V}} \operatorname{dim}(V) \chi_{V}
\end{aligned}
$$

for $a \in \mathbb{R}_{>0}$.

Lemma 5.1. The sum in (5.7) is absolutely convergent for every $a \in \mathbb{R}_{>0}$.

Proof. This follows from [App, Sec.3], which we explain now. Let us fix a maximal torus of $G$ and let $T$ denote its Lie algebra, let $\Lambda^{+} \subset T^{*}$ denote the set of dominant weights and let $(-,-)$ be the inner product on $T^{*}$ induced by the Killing form and $|-|$ the induced norm. We will use that, since $G$ is semisimple, there is a bijection of sets [Kna, Thm. 5.5]

$$
\begin{aligned}
\hat{G} \stackrel{\cong}{\longrightarrow} \Lambda^{+} \\
V \mapsto \lambda_{V}, \\
V_{\lambda} \leftrightarrow \lambda .
\end{aligned}
$$

From [Sug, (1.17)] and [App, (3.2)] we have that (by the Weyl dimension formula) for $V \in \hat{G}$ with dominant weight $\lambda_{V} \in \Lambda^{+}$

$$
\operatorname{dim}(V) \leq N\left|\lambda_{V}\right|^{m},
$$


where $N \in \mathbb{R}_{>0}$ is a constant independent of $V$ and $2 m=\operatorname{dim}(G)-\operatorname{rank}(G)$.

From [Sug, Lem. 1.1] we can express the value of the Casimir element in $V$ using the highest weight $\lambda_{V}$ of $V$ and the half sum of simple roots $\rho$ as

$$
\sigma_{V}=\left(\lambda_{V}, \lambda_{V}+2 \rho\right) \text {. }
$$

It follows directly $[\mathrm{App},(3.5)]$ that

$$
\left|\lambda_{V}\right|^{2} \leq \sigma_{V}
$$

We can give an estimate for the norm of $\lambda_{V}$ as follows. The choice of simple roots gives a bijection $\mathbb{Z}^{\operatorname{rank}(G)} \rightarrow \Lambda^{+}$which we write as $n \mapsto \lambda(n)$. Using the proof of [Sug, Lem. 1.3] there are $C_{1}, C_{2} \in \mathbb{R}_{\geq 0}$ such that for every $n \in \mathbb{Z}^{\operatorname{rank}(G)}$

$$
C_{1}\|n\| \leq|\lambda(n)| \leq C_{2}\|n\|,
$$

where $\|n\|^{2}=\sum_{i=1}^{\operatorname{rank}(G)} n_{i}^{2}$.

Let $b(j)$ denote the number of $n \in \mathbb{Z}^{\operatorname{rank}(G)}$ with $\|n\|^{2}=j$. We can easily give a (very rough) estimate of this by the volume of the $\operatorname{rank}(G)$-dimensional cube with edge length $2 j^{1 / 2}+1$ :

$$
b(j) \leq\left(2 j^{1 / 2}+1\right)^{\operatorname{rank}(G)} .
$$

We compute the squared norm of $\eta_{a}$ following the computation in [App, Ex. 3.4.1].

$$
\begin{aligned}
& \left\|\eta_{a}\right\|^{2}=\sum_{V \in \hat{G}}(\operatorname{dim}(V))^{2} e^{-2 a \sigma_{V}} \stackrel{(5.8)}{=} \sum_{\lambda \in \Lambda^{+}}\left(\operatorname{dim}\left(V_{\lambda}\right)\right)^{2} e^{-2 a \sigma_{V_{\lambda}}} \\
& \stackrel{(5.9)}{\leq} N^{2} \sum_{\lambda \in \Lambda^{+}}|\lambda|^{2 m} e^{-2 a \sigma_{V_{\lambda}}} \stackrel{(5.11)}{\leq} N^{2} \sum_{\lambda \in \Lambda^{+}}|\lambda|^{2 m} e^{-2 a|\lambda|^{2}} \\
& \stackrel{(5.12)}{\leq} N^{2} C_{2}^{2 m} \sum_{n \in \mathbb{Z}^{\operatorname{rank}(G)}}\|n\|^{2 m} e^{-2 a C_{1}\|n\|^{2}}=N^{2} C_{2}^{2 m} \sum_{j=1}^{\infty} b(j) j^{m} e^{-2 a C_{1} j} \\
& \stackrel{(5.13)}{\leq} N^{2} C_{2}^{2 m} \sum_{j=1}^{\infty}\left(2 j^{1 / 2}+1\right)^{\operatorname{rank}(G)} j^{m} e^{-2 a C_{1} j},
\end{aligned}
$$

which converges.

Finally we define the counit as $\varepsilon_{a}:=\eta_{a}^{\dagger}: L^{2}(G) \rightarrow \mathbb{C}$. Explicitly, for $f \in L^{2}(G)$,

$$
\varepsilon_{a}(f)=\left\langle\eta_{a}, f\right\rangle=\sum_{V \in \hat{G}} e^{-a \sigma_{V}} \operatorname{dim}(V) \int_{G} \chi_{V}(x) f\left(x^{-1}\right) d x .
$$

Again for $a \in \mathbb{R}_{>0}$ let

$$
\begin{aligned}
P_{a}: L^{2}(G) & \rightarrow L^{2}(G) \\
f & \mapsto \mu\left(\eta_{a} \otimes f\right),
\end{aligned}
$$

$\mu_{a}:=P_{a} \circ \mu$ and $\Delta_{a}:=\Delta \circ P_{a}$. 
Proposition 5.2. $L^{2}(G)$, together with the family of morphisms $\mu_{a}, \eta_{a}, \Delta_{a}$ and $\varepsilon_{a}$ for $a \in \mathbb{R}_{>0}$ defined above is a strongly separable symmetric $\dagger-R F A$.

Before proving this proposition let us state a lemma. Let $V \in \hat{G}$ and define

$$
\begin{array}{ll}
\mu_{a}^{V}:=\left.\mu_{a}\right|_{M_{V} \otimes M_{V}}, & \eta_{a}^{V}:=e^{-a \sigma_{V}} \operatorname{dim}(V) \chi_{V}, \\
\Delta_{a}^{V}:=\left.\Delta\right|_{M_{V}}, & \varepsilon_{a}^{V}:=\left.\varepsilon_{a}\right|_{M_{V}} .
\end{array}
$$

From a computation using orthogonality of the $f_{i j}^{V}$ we can obtain the following formulas:

$$
\begin{aligned}
P_{a}\left(f_{i j}^{V}\right) & =e^{-a \sigma_{V}} f_{i j}^{V} \in M_{V}, \\
\mu_{a}\left(f_{i j}^{V} \otimes f_{k l}^{V}\right) & =\delta_{j k} e^{-a \sigma_{V}}(\operatorname{dim}(V))^{-1 / 2} f_{i l}^{V} \in M_{V}, \\
\Delta_{a}\left(f_{i j}^{V}\right) & =e^{-a \sigma_{V}}(\operatorname{dim}(V))^{-1 / 2} \sum_{k=1}^{\operatorname{dim}(V)} f_{i k}^{V} \otimes f_{k j}^{V} \in M_{V} \otimes M_{V}, \\
\varepsilon_{a}\left(f_{i j}^{V}\right) & =e^{-a \sigma_{V}}(\operatorname{dim}(V))^{1 / 2} \delta_{i j} .
\end{aligned}
$$

Lemma 5.3. Let $V \in \hat{G}$. Then $M_{V}$ is a strongly separable symmetric $\dagger-R F A$ with the structure maps in (5.17).

Proof. Checking the algebraic relations is a straightforward calculation. As an example, we compute the window element of $M_{V}$.

$$
\begin{aligned}
\mu_{a_{1}}^{V} \circ \Delta_{a_{2}}^{V} \circ \eta_{a_{3}}^{V} & =\sum_{l=1}^{\operatorname{dim}(V)} \mu_{a_{1}}^{V} \circ \Delta_{a_{2}}^{V}\left(f_{l l}^{V}\right) e^{-a_{3} \sigma_{V}}(\operatorname{dim}(V))^{1 / 2} \\
& =\sum_{k, l=1}^{\operatorname{dim}(V)} \mu_{a_{1}}^{V}\left(f_{l k}^{V} \otimes f_{k l}^{V}\right) e^{-\left(a_{2}+a_{3}\right) \sigma_{V}} \\
& =\sum_{k, l=1}^{\operatorname{dim}(V)} f_{l l}^{V} e^{-\left(a_{1}+a_{2}+a_{3}\right) \sigma_{V}}(\operatorname{dim}(V))^{-1 / 2} \\
& =e^{-\left(a_{1}+a_{2}+a_{3}\right) \sigma_{V}} \operatorname{dim}(V) \chi_{V}=\eta_{a_{1}+a_{2}+a_{3}}^{V}
\end{aligned}
$$

which is clearly invertible.

Via the correspondence in Corollary 2.15, the finite dimensional RFA $M_{V}$ is given by the Frobenius algebra $M_{V}$ (with structure maps at $a=0$ ) and the element in the center $\sigma_{V} \cdot \operatorname{id}_{M_{V}}$.

Proof of Proposition 5.2. Let $V \in \hat{G}$ and let us compute the following norms.

$$
\begin{aligned}
\left\|\eta_{a}^{V}\right\|^{2} & =e^{-2 a \sigma_{V}}(\operatorname{dim}(V))^{2}\left\langle\chi_{V}, \chi_{V}\right\rangle=e^{-2 a \sigma_{V}} \operatorname{dim}(V) \sum_{k, l=1}^{\operatorname{dim}(V)}\left\langle f_{k k}^{V}, f_{l l}^{V}\right\rangle \\
& =e^{-2 a \sigma_{V}}(\operatorname{dim}(V))^{2}
\end{aligned}
$$


Let $\varphi=\sum_{i, j=1}^{\operatorname{dim}(V)} \varphi_{i j} f_{i j}^{V} \in M_{V}$ and compute

$$
\left\|\Delta_{a}^{V}(\varphi)\right\|^{2}=e^{-2 a \sigma_{V}}(\operatorname{dim}(V))^{-1} \sum_{i, j, k=1}^{\operatorname{dim}(V)}\left|\varphi_{i j}\right|^{2}\left\|f_{i k}^{V} \otimes f_{k j}^{V}\right\|^{2}=e^{-2 a \sigma_{V}}\|\varphi\|^{2},
$$

so $\left\|\Delta_{a}^{V}\right\|=e^{-a \sigma_{V}}$. Since $M_{V}$ is a $\dagger$-RFA, $\left\|\varepsilon_{a}^{V}\right\|=\left\|\eta_{a}^{V}\right\|$ and $\left\|\mu_{a}^{V}\right\|=\left\|\Delta_{a}^{V}\right\|$.

We now would like to take the direct sum of the RFAs $M_{V}$ for all $V \in \hat{G}$, so we check the conditions of Proposition 2.16: the sum is convergent since it is the squared norm of $\eta_{a} \in L^{2}(G)$ and the supremum is clearly bounded. Therefore $L^{2}(G)$ is an RFA.

Clearly, $L^{2}(G)$ is strongly separable, symmetric and Hermitian by Lemma 5.3.

Now we turn to define an RFA structure on $C l^{2}(G)$.

Proposition 5.4. The centre of $L^{2}(G)$ is $C l^{2}(G)$ and it is a commutative $\dagger-R F A$.

Proof. Let us compute the morphism $D_{a}$ from (4.3). For $\varphi=\sum_{V \in \hat{G}} \sum_{i, j=1}^{\operatorname{dim}(V)} \varphi_{i j}^{V} f_{i j}^{V} \in$ $L^{2}(G)$ we find:

$$
\begin{aligned}
D_{a}(\varphi) & =\mu_{a_{2}} \circ \sigma_{L^{2}(G), L^{2}(G)} \circ \Delta_{a_{1}}(\varphi) \\
& =\mu_{a_{2}} \circ \sigma_{L^{2}(G), L^{2}(G)}\left(\sum_{V \in \hat{G}^{i}} \sum_{i, j, k=1}^{\operatorname{dim}(V)} \varphi_{i j}^{V} f_{i k}^{V} \otimes f_{k j}^{V}\right) e^{-a_{1} \sigma_{V}}(\operatorname{dim}(V))^{-1 / 2} \\
& =\sum_{V \in \hat{G}} \sum_{i, j, k=1}^{\operatorname{dim}(V)} \varphi_{i j}^{V} e^{-a \sigma_{V}}(\operatorname{dim}(V))^{-1} \delta_{i j} f_{k k}^{V} \\
& =\sum_{V \in \hat{G}} \sum_{i=1}^{\operatorname{dim}(V)} \varphi_{i i}^{V} e^{-a \sigma_{V}}(\operatorname{dim}(V))^{-1 / 2} \chi_{V} .
\end{aligned}
$$

From this equation we immediately have that $\left.D_{a}\right|_{C l^{2}(G)}=\left.P_{a}\right|_{C l^{2}(G)}$. We now show that $\left.D_{a}\right|_{\left(C l^{2}(G)\right)^{\perp}}=0$. Using (5.4), we have that $\varphi \in\left(C l^{2}(G)\right)^{\perp} \subset L^{2}(G)$ if and only if for every $W \in \hat{G}\left\langle\chi_{W}, \varphi\right\rangle=0$. We can compute this using the orthogonality relation (5.2) to get the following: $\varphi \in\left(C l^{2}(G)\right)^{\perp}$ if and only if for every $W \in \hat{G}$ we have that $\sum_{k=1}^{\operatorname{dim}(W)} \varphi_{k k}^{W}=0$. By (5.25) we get that $D_{a}(\varphi)=0$.

We have shown that the image of $D_{0}$ is $C l^{2}(G)$, therefore it is the centre of $L^{2}(G)$ by Lemma 4.3. Furthermore it is a $\uparrow$-RFA, since $L^{2}(G)$ is a $\dagger$-RFA and $D_{0}$ is self-adjoint.

For completeness we give the comultiplication $\Delta_{a}^{C l^{2}(G)}$ of $C l^{2}(G)$. For $\varphi=\sum_{V \in \hat{G}}$ $\varphi^{V} \chi_{V} \in C l^{2}(G)$

$$
\Delta_{a}^{C l^{2}(G)}(\varphi)=\sum_{V \in \hat{G}} \varphi^{V} e^{-a \sigma_{V}}(\operatorname{dim}(V))^{-2} \chi_{V} \otimes \chi_{V}
$$

Remark 5.5. Note that for both $L^{2}(G)$ and $C l^{2}(G)$, the $a \rightarrow 0$ limit of the multiplication and comultiplication exists (by definition), but the $a \rightarrow 0$ limit of the unit and counit does not, cf. the proof of Proposition 5.2. 
5.2. State sum construction of $2 d$ Yang-Mills theory. In this section we give state sum data for $2 \mathrm{~d}$ YM theory following [Wit]. The plaquette weights $W_{a}^{k}: \mathbb{C} \rightarrow\left(L^{2}(G)\right)^{\otimes k}$ for $k \in \mathbb{Z}_{\geq 0}$ and $a \in \mathbb{R}>0$ are

$$
W_{a}^{k}(1)\left(x_{1}, \ldots, x_{k}\right)=\sum_{V \in \hat{G}} e^{-a \sigma_{V}} \operatorname{dim}(V) \chi_{V}\left(x_{1} \cdots x_{k}\right),
$$

and the contraction and $\zeta_{a}$ are given by

$$
\beta_{a}:=\left(W_{a}^{2}\right)^{\dagger}, \quad \zeta_{a}:=P_{a}
$$

where $P_{a}$ is as in (5.16). Now we are ready to define $2 \mathrm{~d}$ YM theory, which maps $\mathbb{S}^{1}$ to the centre of $L^{2}(G)$, see Proposition 5.4.

Definition 5.6. The 2-dimensional Yang-Mills (2d YM) theory with gauge group $G$ is the state sum area-dependent QFT

$$
\mathcal{Z}_{\mathrm{YM}}^{G}:=\mathcal{Z}_{L^{2}(G)}: \mathcal{B o r d}_{2}^{\text {area }} \rightarrow \mathcal{H i l b}
$$

of Theorem 4.4. The commutative RFA it assigns to the circle is $\mathcal{Z}_{\mathrm{YM}}^{G}\left(\mathbb{S}^{1}\right)=C l^{2}(G)$.

Next we compute $\mathcal{Z}_{\mathrm{YM}}^{G}$ on connected surfaces with area and $b \geq 0$ boundary components. For $b=0$ the result agrees with [Wit, Eqn. (2.51)] (see also [Rus, Eqn. (27)]).

Proposition 5.7. Let $(\Sigma, a):\left(\mathbb{S}^{1}\right)^{\sqcup b_{\mathrm{in}}} \rightarrow\left(\mathbb{S}^{1}\right)^{\sqcup b_{\text {out }}}$ be a connected bordism of genus $g$ with $b_{\text {in }}$ ingoing and $b_{\text {out }}$ outgoing boundary components and with area $a$. Then for $V_{j} \in \hat{G}$ for $j=1, \ldots, b_{\text {out }}$ we have

$$
\begin{aligned}
& \mathcal{Z}_{\mathrm{YM}}^{G}(\Sigma, a)\left(\chi_{V_{1}} \otimes \cdots \otimes \chi_{b_{b_{\mathrm{in}}}}\right) \\
& = \begin{cases}\sum_{V \in \hat{G}} e^{-a \sigma_{V}(\operatorname{dim}(V))^{\chi(\Sigma)} \cdot\left(\chi_{V}\right)^{\otimes b_{\text {out }}}} & \text { if } b_{\text {in }}=0 \\
e^{-a \sigma_{V_{1}}\left(\operatorname{dim}\left(V_{1}\right)\right)^{\chi(\Sigma)} \cdot\left(\chi_{V_{1}}\right)^{\otimes b_{\text {out }}}} & \text { if } b_{\text {in }} \geq 1 \text { and } V_{1}=\cdots=V_{b_{\text {in }}}, \\
0 & \text { else }\end{cases}
\end{aligned}
$$

where $\chi(\Sigma)=2-2 g-b_{\text {in }}-b_{\text {out }}$ is the Euler characteristic of $\Sigma$. For $b_{\text {in }}=0\left(b_{\text {out }}=0\right)$ the source (the target) is $\mathbb{C}$ and the factors of $\chi_{V}$ or $\chi_{V_{j}}$ are absent.

Proof. We first consider the case that $b:=b_{\text {out }} \geq 1$ and $b_{\text {in }}=0$. Denote the map assigned to the two-holed torus by $\varphi_{a}=\mu \circ\left(\mathrm{id} \otimes\left(\mu \circ \sigma_{L^{2}(G), L^{2}(G)} \circ \Delta\right) \circ \Delta_{a}^{(2)}\right.$ : $L^{2}(G) \rightarrow L^{2}(G)$ and compute

$$
\begin{aligned}
\varphi_{a}\left(f_{i j}^{V}\right) & =\mu \circ\left(\operatorname{id} \otimes\left(\mu \circ \sigma_{L^{2}(G), L^{2}(G)}\right)\left(\sum_{k, l=1}^{\operatorname{dim}(V)} e^{-a \sigma_{V}}(\operatorname{dim}(V))^{-1} f_{i k}^{V} \otimes f_{k l}^{V} \otimes f_{l j}^{V}\right)\right. \\
& =\sum_{k, l=1}^{\operatorname{dim}(V)} e^{-a \sigma_{V}}(\operatorname{dim}(V))^{-2} \delta_{j k} \delta_{k l} f_{i l}^{V}=e^{-a \sigma_{V}}(\operatorname{dim}(V))^{-2} f_{i j}^{V} .
\end{aligned}
$$


Using this, we compute for $a_{0}, \ldots, a_{g+1} \in \mathbb{R}_{>0}$ with $a=\sum_{i=0}^{g+1} a_{i}$ that

$$
\begin{aligned}
\Delta_{a_{g+1}}^{(b)} \circ \prod_{i=1}^{g} \varphi_{a_{i}} \circ \eta_{a_{0}} & =\Delta_{a_{g+1}}^{(b)}\left(\sum_{V \in \hat{G}} e^{-\left(a-a_{g+1}\right) \sigma_{V}}(\operatorname{dim}(V))^{1-2 g} \chi_{V}\right) \\
& =\left[\left(x_{1}, \ldots, x_{b}\right) \mapsto\left(\sum_{V \in \hat{G}} e^{-a \sigma_{V}}(\operatorname{dim}(V))^{1-2 g} \chi_{V}\left(x_{1} \ldots x_{b}\right)\right)\right] .
\end{aligned}
$$

Finally, we need to compose (5.32) with $\pi^{\otimes b}$ to get (5.30), where $\pi: L^{2}(G) \rightarrow C l^{2}(G)$ is the projection onto the image of $D_{0}$. We compute using (5.25):

$$
\begin{aligned}
\pi^{\otimes b}\left(\chi_{V}\left(x_{1} \ldots x_{b}\right)\right) & =\pi^{\otimes b}\left(\sum_{k_{1}, \ldots, k_{b}=1}^{\operatorname{dim}(V)}(\operatorname{dim}(V))^{-b / 2} f_{k_{1} k_{2}}^{V}\left(x_{1}\right) \ldots f_{k_{b} k_{1}}^{V}\left(x_{b}\right)\right) \\
& =(\operatorname{dim}(V))^{1-b} \chi_{V}\left(x_{1}\right) \ldots \chi_{V}\left(x_{b}\right) .
\end{aligned}
$$

For the case $b_{\text {in }}=b_{\text {out }}=0$ we use functoriality. Let $\Sigma^{\prime}$ the surface obtained by cutting out a disk from $\Sigma$. Compose $\mathcal{Z}_{\mathrm{YM}}^{G}\left(\Sigma^{\prime}, a-a^{\prime}\right)$ with $\varepsilon_{a^{\prime}}$ and use (5.21).

For the case $b_{\text {in }} \neq 0$ we need to turn back outgoing boundary components by composing with cylinders with two ingoing boundary components and with area $a$, which we denote with $(C, a)$. For $U, W \in \hat{G}$ we have

$$
\mathcal{Z}_{\mathrm{YM}}^{G}(C, a)\left(\chi_{U}, \chi_{W}\right)=e^{-a \sigma_{U}} \delta_{U, V} .
$$

Using the result for the $b_{\text {in }}=0$ case and (5.34) we get the claimed expression.

Remark 5.8. As already noted in Remark 5.5, $\eta_{a}$ and $\left.\varepsilon_{a}\right|_{C l^{2}(G)}$, i.e. the value of $\mathcal{Z}_{\mathrm{YM}}^{G}$ on a disc with one outgoing (resp. one ingoing) boundary component, do not have zero area limits. On the other hand, the expression (5.30) has a zero area limit if $\chi(\Sigma)=$ $2-2 g-\left(b_{\text {in }}+b_{\text {out }}\right)<0$. Note that this is the condition of $\Sigma$ being stable. Indeed, the $\chi_{V}$ are orthogonal for different $V$ and have norm $\left\|\chi_{V}\right\|=1$, and for a given $\alpha \in \mathbb{Z}$ the sum $\sum_{V \in \hat{G}}(\operatorname{dim}(V))^{\alpha}$ converges if $\alpha \leq-2$. To see this, use the bijection from (5.8) and the estimate from (5.9) to get

$$
\sum_{V \in \hat{G}}(\operatorname{dim}(V))^{\alpha} \leq \sum_{\lambda \in \Lambda^{+}}\left(\operatorname{dim}\left(V_{\lambda}\right)\right)^{\alpha} \leq N \sum_{\lambda \in \Lambda^{+}}|\lambda|^{m \alpha},
$$

which converges for $-m \alpha>\operatorname{rank}(G)$ by [Sug, Lem. 1.3]. Then use that $m=(\operatorname{dim}(G)-$ $\operatorname{rank}(G)) / 2$ and that $3 \operatorname{rank}(G) \leq \operatorname{dim}(G)$ to get $\alpha<-1$, and since $\alpha$ is an integer $\alpha \leq-2$. These limits are related in [Wit] to volumes of moduli spaces of flat connections (see e.g. [KMT] for more results and references). For example for $G=S U$ (2) we have, for $g \geq 2$ and $b_{\text {in }}=b_{\text {out }}=0$,

$$
\lim _{a \rightarrow 0} \mathcal{Z}_{\mathrm{YM}}^{S U(2)}(\Sigma, a)(1)=\sum_{n=1}^{\infty} n^{-2 g+2}=\zeta(2 g-2),
$$

where $\zeta$ is the Riemann zeta-function. For general $G$, these functions are also referred to as Witten zeta-functions, see e.g. [KMT]. 
Acknowledgement. The authors thank Yuki Arano, Nils Carqueville, Alexei Davydov, Reiner Lauterbach, Pau Enrique Moliner, Chris Heunen, André Henriques, Ehud Meir, Catherine Meusburger, Gregor Schaumann, Richard Szabo and Stefan Wagner for helpful discussions and comments. We also thank the referees for their detailed comments which significantly improved the exposition of this paper. LS is supported by the DFG Research Training Group 1670 "Mathematics Inspired by String Theory and Quantum Field Theory".

Funding Open access funding provided by Institute of Science and Technology (IST Austria).

Open Access This article is licensed under a Creative Commons Attribution 4.0 International License, which permits use, sharing, adaptation, distribution and reproduction in any medium or format, as long as you give appropriate credit to the original author(s) and the source, provide a link to the Creative Commons licence, and indicate if changes were made. The images or other third party material in this article are included in the article's Creative Commons licence, unless indicated otherwise in a credit line to the material. If material is not included in the article's Creative Commons licence and your intended use is not permitted by statutory regulation or exceeds the permitted use, you will need to obtain permission directly from the copyright holder. To view a copy of this licence, visit http://creativecommons.org/licenses/by/4.0/.

Publisher's Note Springer Nature remains neutral with regard to jurisdictional claims in published maps and institutional affiliations.

\section{A Proof of Joint Continuity}

The following lemma will be instrumental in showing that various joint continuity conditions hold automatically in Hilb. A similar statement can be found in [KMD, Sec. 2].

Lemma A.1. Let $\mathcal{H}_{i} \in \mathcal{H}$ ilb $(i=1,2)$. Let $X$ be a subset of a finite-dimensional normed vector space (e.g. $X=\mathbb{R}_{\geq 0}$ ). Equip $X$ with the induced topology and let $a \mapsto S_{a}^{(i)}$ be two continuous maps $X \rightarrow \mathcal{B}\left(\mathcal{H}_{i}\right)$. Then the map $X^{2} \rightarrow \mathcal{B}\left(\mathcal{H}_{1} \otimes \mathcal{H}_{2}\right),(a, b) \mapsto S_{a}^{(1)} \otimes S_{b}^{(2)}$ is jointly continuous.

Proof. We will first show that the map $a \mapsto\left\|S_{a}^{(i)}\right\|$ is bounded on compact subsets of $X$. Let $K \subset X$ be compact. By strong continuity we have that for every $h \in \mathcal{H}_{i}$ the map $a \mapsto S_{a}^{(i)}(h)$ is continuous, so in particular the map $a \mapsto\left\|S_{a}^{(i)}(h)\right\|$ is continuous, hence bounded on $K$. By the Uniform Boundedness Principle [Con1, Ch.III.14] the map $a \mapsto\left\|S_{a}^{(i)}\right\|$ is bounded on $K$.

Now we turn to the claim in the lemma. Let $a_{0}, b_{0} \in X$ and $\kappa, \varepsilon \in \mathbb{R}_{>0}$ be fixed. We will show that the map $(a, b) \mapsto S_{a}^{(1)} \otimes S_{b}^{(2)}$ is continuous at $\left(a_{0}, b_{0}\right)$.

For $T \in \mathcal{H}_{1} \otimes \mathcal{H}_{2}$ take a sequence $\left\{T_{n}\right\}_{n}$ in the algebraic tensor product of $\mathcal{H}_{1}$ and $\mathcal{H}_{2}$ such that $T_{n} \stackrel{n \rightarrow \infty}{\longrightarrow} T$. We have the estimate

$$
\begin{aligned}
& \left\|\left(S_{a}^{(1)} \otimes S_{b}^{(2)}-S_{a_{0}}^{(1)} \otimes S_{b_{0}}^{(2)}\right) T\right\| \\
& \quad \leq\left\|\left(S_{a}^{(1)} \otimes S_{b}^{(2)}-S_{a_{0}}^{(1)} \otimes S_{b_{0}}^{(2)}\right)\right\| \cdot\left\|T-T_{n}\right\|+\left\|\left(S_{a}^{(1)} \otimes S_{b}^{(2)}-S_{a_{0}}^{(1)} \otimes S_{b_{0}}^{(2)}\right) T_{n}\right\| .
\end{aligned}
$$

We give an estimate for the first term on the right hand side of (A.1). Fix some $\delta_{1}>0$. Then by the above boundedness result there is a $\kappa>0$ such that for every $a, b \in X$ with $\left|a-a_{0}\right|+\left|b-b_{0}\right|<\delta_{1}$ we have $\left\|S_{a}^{(1)}\right\|<\kappa$ and $\left\|S_{b}^{(2)}\right\|<\kappa$. So we have

$$
\begin{aligned}
\left\|S_{a}^{(1)} \otimes S_{b}^{(2)}-S_{a_{0}}^{(1)} \otimes S_{b_{0}}^{(2)}\right\| & \leq\left\|S_{a}^{(1)}\right\| \cdot\left\|S_{b}^{(2)}\right\|+\left\|S_{a_{0}}^{(1)}\right\| \cdot\left\|S_{b_{0}}^{(2)}\right\| \\
& \leq \kappa^{2}+\left\|S_{a_{0}}^{(1)}\right\| \cdot\left\|S_{b_{0}}^{(2)}\right\|=: N_{a_{0}, b_{0}}^{\kappa} .
\end{aligned}
$$


Since $T_{n} \stackrel{n \rightarrow \infty}{\longrightarrow} T$, we can choose $n$ (which we keep fixed from now on) such that

$$
\left\|T-T_{n}\right\|<\frac{\varepsilon}{2 N_{a_{0}, b_{0}}^{\kappa}} .
$$

Putting (A.2) and (A.3) together we get

$$
\left\|\left(S_{a}^{(1)} \otimes S_{b}^{(2)}-S_{a_{0}}^{(1)} \otimes S_{b_{0}}^{(2)}\right)\right\| \cdot\left\|T-T_{n}\right\| \leq \frac{\varepsilon}{2} .
$$

We give an estimate for the last term in (A.1). Recall that each $T_{n}$ was chosen in the algebraic tensor product of $\mathcal{H}_{1}$ and $\mathcal{H}_{2}$. Thus $T_{n}$ is a finite sum of elementary tensors,

$$
T_{n}=\sum_{j=1}^{t_{n}} x_{n}^{j} \otimes y_{n}^{j}
$$

for $t_{n} \in \mathbb{Z}_{\geq 1}, x_{n}^{j} \in \mathcal{H}^{(1)}$ and $y_{n}^{j} \in \mathcal{H}^{(2)}$. Using this, we get:

$$
\begin{aligned}
& \left\|\left(S_{a}^{(1)}-S_{a_{0}}^{(1)}\right) \otimes S_{b}^{(2)} T_{n}+S_{a_{0}}^{(1)} \otimes\left(S_{b}^{(2)}-S_{b_{0}}^{(2)}\right) T_{n}\right\| \\
& \quad \leq \sum_{j=1}^{t_{n}}\left(\left\|\left(S_{a}^{(1)}-S_{a_{0}}^{(1)}\right) x_{n}^{j}\right\| \cdot\left\|S_{b}^{(2)}\right\| \cdot\left\|y_{n}^{j}\right\|+\|\left(S_{a_{0}}^{(1)}\|\cdot\| x_{n}^{j}\|\cdot\|\left(S_{b}^{(2)}-S_{b_{0}}^{(2)}\right) y_{n}^{j} \|\right) .\right.
\end{aligned}
$$

By strong continuity of $a \mapsto S_{a}^{(i)}$ we can chose $\delta_{2}>0$ such that for every $a, b \in X$ with $\left|a-a_{0}\right|+\left|b-b_{0}\right|<\delta_{2}$ we have

$$
\left\|\left(S_{a}^{(1)}-S_{a_{0}}^{(1)}\right) x_{n}^{j}\right\|<\frac{\varepsilon}{4 t_{n} \kappa\left\|y_{n}^{j}\right\|} \quad \text { and } \quad\left\|\left(S_{b}^{(2)}-S_{b_{0}}^{(2)}\right) y_{n}^{j}\right\|<\frac{\varepsilon}{4 t_{n}\left\|S_{a_{0}}^{(1)}\right\| \cdot\left\|x_{n}^{j}\right\|} \text {, }
$$

for every $j=1, \ldots, t_{n}$, since these are only finitely many conditions to satisfy. Let $\delta:=\min \left\{\delta_{1}, \delta_{2}\right\}$. Then for every $a, b \in X$ with $\left|a-a_{0}\right|+\left|b-b_{0}\right|<\delta$ we have that

$$
\left\|\left(S_{a}^{(1)} \otimes S_{b}^{(2)}-S_{a_{0}}^{(1)} \otimes S_{b_{0}}^{(2)}\right) T_{n}\right\| \leq \frac{\varepsilon}{2} .
$$

Finally, using (A.4) and (A.8) we have that

$$
\left\|\left(S_{a}^{(1)} \otimes S_{b}^{(2)}-S_{a_{0}}^{(1)} \otimes S_{b_{0}}^{(2)}\right) T\right\|<\varepsilon .
$$

Proof of Lemma 2.5. Let $\varphi_{a_{1}, \ldots, a_{N}}: A^{\otimes n} \rightarrow A^{\otimes m}$ be a morphism in Hilb tensor generated by $\mu_{a}, \eta_{a}, \Delta_{a}$ and $\varepsilon_{a}$, involving a total of $N$ connected components when drawing it using the graphical calculus, with parameters $a_{1}, \ldots, a_{N}$. One can write $\varphi_{a_{1}, \ldots, a_{N}}$ in the form $\varphi_{\varepsilon_{1}}^{(1)} \circ\left(\bigotimes_{i=1}^{N} P_{a_{i}-\varepsilon_{1}-\varepsilon_{2}}\right) \circ \varphi_{\varepsilon_{2}}^{(2)}$ for some $\varepsilon_{1}, \varepsilon_{2} \in \mathbb{R}_{>0}$ and morphisms $\varphi_{\varepsilon_{1}}^{(1)}$ and $\varphi_{\varepsilon_{2}}^{(2)}$. Then by separate continuity of the composition of $\mathcal{H i l b}$ and joint continuity of $\bigotimes_{i=1}^{N} P_{a_{i}}$ by Lemma A.1 the morphism $\varphi_{a_{1}, \ldots, a_{N}}$ is jointly continuous in the parameters $a_{1}, \ldots, a_{N}$. 


\section{References}

[Abr] Abrams, L.: Two dimensional topological quantum field theories and Frobenius algebras. J. Knot Theor. Ramif. 5, 569-587 (1996)

[App] Applebaum, D.: Infinitely divisible central probability measures on compact Lie groupsregularity, semigroups and transition kernels. Ann. Probab. 39(6), 2474-2496 (2011). arXiv:1006.4711 [math.PR]

[Ban] Banyaga, A.: Formes-volume sur les variétés à bord. Enseignement Math. 2, 127-131 (1974)

[Bar] Bartlett, B.H.: Categorical Aspects of Topological Quantum Field Theories. PhD thesis, Utrecht University (2005) arXiv:math/0512103 [math.QA]

[BB] Radjavi, H., Rosenthal, P.: Simultaneous Triangularization. Universitext. Springer-Verlag, New York (1994)

[BP] Bachas, C., Petropoulos, P.M.S.: Topological models on the lattice and a remark on string theory cloning. Commun. Math. Phys. 152, 191-202 (1993). arXiv:hep-th/9205031

[Bru] Brunner, I.: Area preserving symmetry in two-dimensional quantum field theory. BONN-IB-9536, diploma thesis (1995)

[Car] Carqueville, N.: Lecture notes on 2-dimensional defect TQFT. Banach Cent. Publ. 114, 49-84 (2018). arXiv:1607.05747 [math.QA]

[CMR] Cordes, S., Moore, G., Ramgoolam, S.: Lectures on 2D Yang-Mills theory, equivariant cohomology and topological field theories. Nucl. Phys. Proc. Suppl. 41, 184-244 (1995). arXiv:hep-th/9411210

[Con1] Conway, J.B.: A Course in Functional Analysis. Graduate Texts in Mathematics, vol. 96. Springer, Berlin (1994)

[Con2] Conway, J.B.: A Course in Operator Theory. Graduate Studies in Mathematics, vol. 21. American Mathematical Society, Providence (2000)

[CR] Carqueville, N., Runkel, I.: Introductory lectures on topological quantum field theory. arXiv:1705.05734 [math.QA]

[CTS] Cunha, B.G.C., Teotonio-Sobrinho, P.: Quasi-topological field theories in two dimensions as soluble models. Int. J. Mod. Phys. A 13, 3667-3690 (1998). arXiv:hep-th/9703014

[Dav] Davydov, A.: Centre of an algebra. Adv. Math. 225(1), 319-348 (2010). arXiv:0908.1250 [math.CT]

[Dij] Dijkgraaf, R.H.: A geometrical approach to two-dimensional conformal field theory. PhD thesis, Utrecht University (1989)

[DKR] Davydov, A., Kong, L., Runkel, I.: Field theories with defects and the centre functor. In: Sati, H., Schreiber, U. (eds.) Mathematical Foundations of Quantum Field and Perturbative String Theory. AMS, Providence (2011). arXiv:1107.0495 [math.QA]

[EN] Engel, K.J., Nagel, R.: One-Parameter Semigroups for Linear Evolution Equations. Graduate Texts in Mathematics. Springer, New York (1999)

[FHK] Fukuma, M., Hosono, S., Kawai, H.: Lattice topological field theory in two dimensions. Commun. Math. Phys. 161, 157-175 (1994). arXiv:hep-th/9212154

[Kan] Kanzaki, T.: Special type of separable algebras over a commutative ring. Proc. Jpn. Acad. 40, 781-786 (1964)

[Kir] Kirillov Jr., A.: On piecewise linear cell decompositions. Algebr. Geom. Topol. 12, 95-108 (2012). arXiv:1009.4227 [math.GT]

[KMD] Khalil, R., Al-Mirbati, R., Drissi, D.: Tensor product semigroups. Eur. J. Pure Appl. Math. 3(5), 881-898 (2010)

[KMT] Komori, Y., Matsumoto, K., Tsumura, H.: Zeta-functions of weight lattices of compact connected semisimple Lie groups. Siauliai Math. Semin. 18, 149-179 (2015). arXiv:1011.0323 [math.NT]

[Kna] Knapp, A.W.: Lie Groups Beyond an Introduction. Progress in Mathematics. Birkhäuser, Boston (2002)

[Koc] Kock, J.: Frobenius Algebras and 2D Topological Quantum Field Theories. Cambridge University Press, Cambridge (2004)

[KR] Kadison, R.V., Ringrose, J.R.: Fundamentals of the Theory of Operator Algebras: Elementary Theory, vol. I. Academic Press, New York (1983)

[Kub] Kubrusly, C.S.: Elements of Operator Theory. Springer, Berlin (2001)

[LP] Lauda, A.D., Pfeiffer, H.: State sum construction of two-dimensional open-closed topological quantum field theories. J. Knot Theor. Ramif. 16, 1121-1163 (2007). arXiv:math/0602047 [math.QA]

[Mig] Migdal, A.A.: Recursion equations in gauge field theories. Sov. Phys. JETP 42, 413 (1975)

[Mos] Moser, J.: On the volume elements on a manifold. Trans. Am. Math. Soc. 120, 286-294 (1965)

[Pie] Pierce, R.S.: Associative Algebras. Graduate Texts in Mathematics, vol. 88. Springer, Berlin (1982) 
[RS] Runkel, I., Szegedy, L.: Topological field theory on r-spin surfaces and the Arf invariant. arXiv:1802.09978 [math.QA]

[Rus] Rusakov, B.E.: Loop averages and partition functions in U(N) gauge theory on two-dimensional manifolds. Mod. Phys. Lett. A 5, 693 (1990)

[Seg] Segal, G.: Topological Field Theory ('Stanford Notes'). Lecture notes from ITP Workshop on Geometry and Physics (1999)

[Sel] Selinger, P.: Dagger compact closed categories and completely positive maps. Electron. Notes Theor. Comput. Sci. 170, 139-163 (2007)

[Sug] Sugiura, M.: Fourier series of smooth functions on compact Lie groups. Osaka J. Math. 8, 33-47 (1971)

[Sze] Szegedy, L.: State-Sum Construction of Two-Dimensional Functorial Field Theories. PhD thesis, Universität Hamburg (2018). http://ediss.sub.uni-hamburg.de/volltexte/2018/9321/

[Tur] Turaev, V.G.: Quantum Invariants of Knots and 3-Manifolds. de Gruyter, Berlin (1994)

[Wit] Witten, E.: On quantum gauge theories in two dimensions. Commun. Math. Phys. 141, 153-209 (1991)

Communicated by Y. Kawahigashi 